\title{
Comparative analysis of Faecalibacterium prausnitzii genomes shows a high level of genome plasticity and warrants separation into new species-level taxa
}

Cormac Brian Fitzgerald ${ }^{1,4+}$, Andrey N. Shkoporov ${ }^{1+}$ D, Thomas D. S. Sutton ${ }^{1}$, Andrei V. Chaplin ${ }^{2}$, Vimalkumar Velayudhan ${ }^{1}$, R. Paul Ross ${ }^{1,3,4}$ and Colin Hill ${ }^{1,3,4^{*}}$

\begin{abstract}
Background: Faecalibacterium prausnitzii is a ubiquitous member of the human gut microbiome, constituting up to $15 \%$ of the total bacteria in the human gut. Substantial evidence connects decreased levels of $F$. prausnitzii with the onset and progression of certain forms of inflammatory bowel disease, which has been attributed to its antiinflammatory potential. Two phylogroups of $F$. prausnitzii have been identified, with a decrease in phylogroup I being a more sensitive marker of intestinal inflammation. Much of the genomic and physiological data available to date was collected using phylogroup II strains. Little analysis of F. prausnitzii genomes has been performed so far and genetic differences between phylogroups I and II are poorly understood.

Results: In this study we sequenced 11 additional F. prausnitzii genomes and performed comparative genomics to investigate intraspecies diversity, functional gene complement and the mobilome of 31 high-quality draft and complete genomes. We reveal a very low level of average nucleotide identity among $F$. prausnitzii genomes and a high level of genome plasticity. Two genomogroups can be separated based on differences in functional gene complement, albeit that this division does not fully agree with separation based on conserved gene phylogeny, highlighting the importance of horizontal gene transfer in shaping F. prausnitzii genomes. The difference between the two genomogroups is mainly in the complement of genes associated with catabolism of carbohydrates (such as a predicted sialidase gene in genomogroup I) and amino acids, as well as defense mechanisms.
\end{abstract}

Conclusions: Based on the combination of ANI of genomic sequences, phylogenetic analysis of core proteomes and functional differences we propose to separate the species F. prausnitzii into two new species level taxa: $F$. prausnitzii sensu stricto (neotype strain $A 2-165^{\top}=$ DSM $17677^{\top}=J C M ~ 31915^{\top}$ ) and F. moorei sp. nov. (type strain ATCC $27768^{\top}=$ NCIMB $13872^{\top}$ ).

Keywords: Faecalibacterium prausnitzii, Ruminococcaceae, Human gut microbiota, IBD, Genome shuffling

\footnotetext{
* Correspondence: c.hill@ucc.ie

${ }^{+}$Cormac Brian Fitzgerald and Andrey N. Shkoporov contributed equally to

this work.

${ }^{1}$ APC Microbiome Ireland, University College Cork, Cork, Ireland

${ }^{3}$ Department of Food Biosciences, Teagasc Food Research Centre,

Moorepark, Fermoy, Ireland

Full list of author information is available at the end of the article
}

(c) The Author(s). 2018 Open Access This article is distributed under the terms of the Creative Commons Attribution 4.0 International License (http://creativecommons.org/licenses/by/4.0/), which permits unrestricted use, distribution, and reproduction in any medium, provided you give appropriate credit to the original author(s) and the source, provide a link to the Creative Commons license, and indicate if changes were made. The Creative Commons Public Domain Dedication waiver (http://creativecommons.org/publicdomain/zero/1.0/) applies to the data made available in this article, unless otherwise stated. 


\section{Background}

Faecalibacterium prausnitzii is a Gram-stain negative, non-sporeforming, acetate-consuming and butyrate-producing, extremely oxygen-sensitive (EOS) member of the Ruminococcaceae family (phylum Firmicutes). The type strain of the species was originally isolated from human faeces in the 1970's and classified as Fusobacterium prausnitzii [1]. Two decades later with the aid of $16 \mathrm{~S}$ rRNA sequence information it was re-assigned to the Clostridium leptum group (clostridial cluster IV [2]). Finally, it was classified as a separate genus named Faecalibacterium (family Ruminococcaceae) in 2002 by Duncan et al. [3]. The importance of the species to human health was not fully realized until the mid-2000's when high throughput sequencing of 16S rRNA libraries and metagenomic analysis of faecal DNA revealed that F. prausnitzii is one of the most abundant bacteria in the human gut, accounting for $5-15 \%$ of the total bacterial population [4-8]. At the same time, decreased $F$. prausnitzii levels were observed in various forms of inflammatory bowel disease (IBD) such as Crohn's disease (CD) and ulcerative colitis (UC), as well as in colorectal cancer (CRC) and type 2 diabetes [8-11].

Over the last decade a substantial number of studies have linked decreased levels of $F$. prausnitzii with the onset and progression of certain forms of IBD [9, 12-16]. Although the connection between $F$. prausnitzii levels and disease activity in UC and pouchitis is controversial, depletion of $F$. prausnitzii in CD, especially in disease flares and in the ileal form of $\mathrm{CD}$, has been demonstrated in both faecal and biopsy samples using a variety of methods (16S rRNA library sequencing, qPCR, RT-qPCR, DNA microarrays, FISH; [8]. It was shown that various anti-inflammatory and anti-bacterial treatments effective in patients with $\mathrm{CD}$, including high-dose cortisol, infliximab, interferon- $\alpha 2 b$, and rifaximin were able to restore normal levels of F. prausnitzii [17-19]. Therefore, it was proposed that the depletion of $F$. prausnitzii is not a causative event in $\mathrm{CD}$, but rather a consequence of mucosal inflammation that generates excessive amounts of reactive oxygen species (ROS). This leads to a significant reduction of mucosa-associated and luminal EOS cultures, including F. prausnitzii [8]. However, this simplified model is argued against by a number of studies that have demonstrated direct anti-inflammatory effects of live F. prausnitzii cells and cell components in both cell culture and animal models of intestinal inflammation [9, 20-28].

Butyric acid is one of the main metabolic end-products of $F$. prausnitzii fermentation. Microbiota-derived butyric acid is known to possess anti-inflammatory activity [29], as well as to serve as one of the main energy sources for colonocytes [30]. In addition, F. prausnitzii has been shown to produce a number of substances with proven anti-inflammatory properties, including a $15 \mathrm{kDa}$ proteinaceous "microbial anti-inflammatory molecule" (MAM) that was able to inhibit the NF- $\mathrm{kB}$ pathway in intestinal epithelial cells and prevent colitis in a murine IBD model [23, 24, 28]. A capsule-like extracellular polymeric matrix has also been shown to suppress the inflammatory response in cultured dendritic cells and alleviate intestinal inflammation in a murine model of IBD [21].

Surprisingly, despite the potential benefits of F. prausnitzii for human health, its genome organization, diversity and the genetic traits associated with the ability to colonize and persist in the human gut have received relatively little attention. As of June 2018, genomes of 21 isolates of $F$. prausnitzii (not including genomes reconstructed from metagenomic assemblies) were available from public databases with variable levels of assembly and annotation quality (https://www.ncbi.nlm.nih.gov/ genome/genomes/682). Of these only two were complete (strains A2-165 and Indica), while the rest were represented by draft assemblies.

Several studies have attempted to separate F. prausnit$z i i$ isolates into subspecies-level groups mainly based on physiological properties [31], 16S rRNA gene sequences $[14,16,31-33]$ and more recently on full genome sequences [34]. Despite some discrepancies between data published by different groups in relation to how many phylogroups of $F$. prausnitzii are present (either 2 [31] or 3 phylogroups [34]), the two main $16 \mathrm{~S}$ rRNA phylogroups that have been proposed, each demonstrating specific and different responses in various gut disorders. Phylogroup I depletion was detected in CD, UC, and CRC, whereas phylogroup II was only decreased in CD [16]. In addition, an overall reduction of mucosa-associated $F$. prausnitzii counts and decreased richness of $16 \mathrm{~S}$ rRNA $F$. prausnitzii phylotypes was observed in both IBD and CRC [14]. Based on these observations several potentially useful indices were proposed for differential diagnosis of several clinical forms of IBD and CRC, which included total F. prausnitzii counts, separate counts for both phylogroups, as well as the Faecalibacterium/Escherichia ratio [35]. A study conducted with $F$. prausnitzii strain A2-165, belonging to phylogroup II has shown that, despite its extreme oxygen sensitivity, the strain could actually benefit from very low oxygen concentrations that it can use for $\mathrm{NADH}$ regeneration through an extracellular electron shuttle [36]. This can partly explain why phylogroup II $F$. prausnitzii seem to be more resistant to oxidative stress in the gut in IBD and CRC. Another recent study has revealed a high level of genome plasticity in F. prausnitzii and an apparently open type of species pangenome [34], which is in line with the observed high level of functional diversity and specialization of $F$. prausnitzii strains.

It is important to note that many of the experimental and in silico studies to investigate mechanisms of 
anti-inflammatory activity, oxygen sensitivity, metabolism and interaction with the host and other bacteria in the gut have been conducted with a single $F$. prausnitzii strain - A2-165. This strain belongs to phylogroup II $[9,22,24,36-38]$ and has apparently been chosen due to its relative ease of growth on culture media and relatively higher resistance to oxygen. Much less data have been gathered so far regarding representatives of phylogroup I. To our knowledge, little effort has been directed towards understanding the genomic differences between the two phylogroups and linking any differences to strain physiology and their impact on human health.

Here we report on genome sequencing of an additional 11 human isolates of $F$. prausnitzii, we describe the genome structure and diversity of this species, and investigate genomic traits underlying the physiological differences between the two previously described phylogroups.

\section{Results}

General characteristics of $F$. prausnitzii genome structure In total, draft genome assemblies were generated for 11 F. prausnitzii strains. Of these 11, nine were novel isolates from six healthy adult individuals (APC942/8-14-2, APC942/18-1, APC942/30-2, APC942/32-1, APC922/ 41-1, APC923/51-1, APC923/61-1, APC924/119 and APC918/95b). Two additional strains, including the original type strain of $F$. prausnitzii [1] were obtained from the ATCC culture collection (ATCC 27766 and ATCC $27768^{\mathrm{T}}$ ). The nine new isolates were chosen from a collection of 184 faecal bacterial strains isolated anaerobically from 6 clinically healthy individuals aged 23-54 years. Strains of $F$. prausnitzii were selected based on partial 16S rRNA gene sequencing (data not shown). The same 16S rRNA sequences allowed us to classify the strains into the two phylogroups suggested earlier $[16,31]$. One additional strain sequence (APC924/74), which we initially identified as $F$. prausnitzii, was in fact a member of the closely related Gemmiger/Subdoligranulum cluster $[39,40]$. That strain was excluded from $F$. prausnitzii comparative genome analysis but retained for comparative analysis at the level of the family Ruminococcaceae. After our initial examination of draft genome assemblies, one representative from each $F$. prausnitzii phylogroup was chosen for further long read sequencing to obtain complete circular chromosomes as described in the Materials and Methods. In addition, we recruited 20 complete and high quality draft genome sequences from 25 available GenBank $F$. prausnitzii genome entries, choosing those which had been assembled into less than 250 contigs and having combined length of at least $2.5 \mathrm{Mbp}$.

Altogether, the genome size of $F$. prausnitzii varied from 2.68 to $3.42 \mathrm{Mbp}$ with a $\mathrm{G}+\mathrm{C}$ content over a wide range of $54.9-63.0 \mathrm{~mol} \%$. The four available complete circular genomes ranged in size from 2.83 to $3.11 \mathrm{Mbp}$ with a $G+C$ content of $56.3-57.2 \mathrm{~mol} \%$ (Table 1). No circular plasmids or other circular extra-chromosomal elements were detected in any of the strains. Circular maps of the two complete $F$. prausnitzii chromosomes sequenced in this study are shown in Figs. 1 and 2. These include the complete genome of APC918/95b the first representative of $F$. prausnitzii phylogroup I to be assembled into a complete genome.

Alignment of the four completed genomes of F. prausnitzii using the Mauve algorithm revealed a strikingly low level of genomic synteny. Only $14 \pm 0.05 \%$ of the genome sequence was located in syntenic, locally collinear blocks (LCBs). For comparison, similarly sized genomes of other diverse bacterial species from the human gut (Clostridiodes difficile, Bacteroides fragilis, Escherichia coli) had between 76 and $83 \%$ of their sequences in syntenic LCBs (Fig. 3, Additional file 1: Figure S1).

In addition to that, alignment of phylogroups I and II genomes, using $942 / 30-2$ and $918 / 95 b$ as references, revealed the presence of numerous genomic islands identified by a low level of homology to other genomes (representatives of both phylogroup I and II), atypical G $+\mathrm{C}$ content and GC skew distribution (Figs. 1 and 2, rings $8-16$ ). In a large number of cases these genomic islands were co-localized with or flanked by genes coding for integrases, site-specific recombinases, plasmidlike replication and mobilization proteins (Figs. 1 and 2, ring 17), but not necessarily with incomplete and remnant prophage elements (Figs. 1 and 2 ring 3 ).

\section{Intraspecies diversity of $F$. prausnitzii and their} phylogenetic position within the family Ruminococcaceae In order to further investigate intraspecies genomic diversity of $F$. prausnitzii we performed average nucleotide identity (ANI) analysis of genomic sequences based on pairwise reciprocal genome-wide BLAST searches. The median level of reciprocal ANI within the species was $84.1 \%$ (with only $52.8 \%$ of median reciprocal sequence coverage by BLAST searches). This was well below the cut-off level of 95\% ANI suggested to define a bacterial species [41, 42]. F. prausnitzii genomes were seen to cluster into several groups when ANI data was subjected to hierarchical clustering. Genomes within these clusters had levels of homology between 94 and 100\% (with reciprocal coverage levels of $60-100 \%$ ). These included a large group of strains belonging to the previously established 16S rRNA phylogoroup I [31], as well as several tight groups of strains, with low levels of ANI between them, together comprising what was earlier established as phylogroup II (Fig. 4, Additional file 2: Figure S2).

In order to establish the phylogenetic position for $F$. prausnitzii and its closest related species within the family Ruminococcaceae, as well as to identify potentially 
Table 1 F. prausnitzii strains used in the study

\begin{tabular}{|c|c|c|c|c|c|c|c|}
\hline Strain name & Coverage, $X$ & Isolation source & Genbank accession & Contigs, $\mathrm{N}$ & Length, Mb & $\mathrm{G}+\mathrm{C}, \mathrm{mol} \%$ & Reference \\
\hline APC942/8-14-2 & 860 & Faeces of 34 y.o. male & PRKZ00000000 & 28 & 2.69 & 57 & Current study \\
\hline APC942/18-1 & 285 & Faeces of 34 y.o. male & PRLA00000000 & 26 & 2.80 & 57.3 & Current study \\
\hline APC $942 / 30-2^{a}$ & 722 & Faeces of 34 y.o. male & СР026548.1 & 1 & 2.83 & 57.2 & Current study \\
\hline APC942/32-1 & 738 & Faeces of 34 y.o. male & PRLB00000000 & 52 & 2.99 & 57.4 & Current study \\
\hline APC922/41-1 & 726 & Faeces of 30 y.o. male & PRLC00000000 & 54 & 2.79 & 57.7 & Current study \\
\hline APC923/51-1 & 783 & Faeces of 30 y.o. male & PRLD00000000 & 70 & 3.03 & 56.2 & Current study \\
\hline APC923/61-1 & 240 & Faeces of 55 y.o. male & PRLE00000000 & 19 & 2.68 & 57.4 & Current study \\
\hline APC918/95b & 349 & Faeces of 25 y.o. female & NZ_CP030777.1 & 1 & 2.97 & 56.4 & Current study \\
\hline APC924/119 & 290 & Faeces of 44 y.o. female & PRLF00000000 & 83 & 3.02 & 56.4 & Current study \\
\hline ATCC 27766 & 950 & human faeces & PXUQ00000000 & 83 & 3.02 & 56.5 & [1] \\
\hline ATCC $27768^{\top}$ & 300 & human faeces & PXUP00000000 & 76 & 3.03 & 56.4 & [1] \\
\hline CNCM 4540 & 1369 & human faeces & NZ_NMTQ00000000.1 & 48 & 3.04 & 55.7 & [34] \\
\hline CNCM 4541 & 1403 & human faeces & NZ_NMTR00000000.1 & 78 & 2.82 & 58.1 & [34] \\
\hline CNCM 4542 & 1401 & human faeces & NZ_NMTS000000000.2 & 106 & 2.91 & 55.8 & [34] \\
\hline CNCM 4543 & 1645 & human faeces & NZ_NMTT000000000.2 & 22 & 3.08 & 56.2 & [34] \\
\hline CNCM 4544 & 1627 & human faeces & NZ_NMTU00000000.1 & 69 & 2.80 & 56.0 & [34] \\
\hline CNCM 4546 & 2019 & human faeces & NZ_NMTV00000000.1 & 244 & 3.42 & 54.9 & [34] \\
\hline CNCM 4573 & 1319 & human faeces & NZ_NMTW00000000.1 & 83 & 3.28 & 55.9 & {$[34]$} \\
\hline CNCM 4574 & 1821 & human faeces & NZ_NMTX000000000.1 & 38 & 3.09 & 56.3 & [34] \\
\hline CNCM 4575 & 1798 & human faeces & NZ_NMTY00000000.1 & 37 & 3.01 & 57.5 & [34] \\
\hline CNCM 4644 & 1298 & human faeces & NZ_NMTZ000000000.1 & 36 & 2.92 & 56.4 & [34] \\
\hline$S L 3 / 3$ & 20 & Faeces of 46 y.o. female & FP929046.1 & 1 & 3.21 & 55.7 & [31] \\
\hline L2-6 & 29 & Faeces of 2 y.o. male & FP929045.1 & 1 & 3.32 & 56.8 & [3] \\
\hline $\mathrm{M} 21 / 2$ & 8.4 & Faeces of 36 y.o. female & NZ_ABED00000000.2 & 29 & 3.13 & 56.2 & [31] \\
\hline A2-165 & 395 & Faeces of 34 y.o. female & NZ_CP022479.1 & 1 & 3.11 & 56.3 & [3] \\
\hline KLE1255 & 65 & N/A & NZ_AECU00000000.1 & 249 & 2.93 & 56.3 & Weinstock et al. $2010^{\mathrm{b}}$ \\
\hline 2789STDY5608869 & N/A & human faeces & NZ_CYYL00000000.1 & 49 & 2.85 & 57.7 & [73] \\
\hline 2789STDY5834970 & N/A & human faeces & NZ_CYXN00000000.1 & 86 & 3.05 & 56.0 & [73] \\
\hline Indica & 500 & human faeces & NZ_CP023819.1 & 1 & 2.87 & 56.9 & {$[56]$} \\
\hline AHMP_21 & 203 & human faeces & NZ_NOUV00000000.1 & 85 & 3.02 & 57.4 & [34] \\
\hline HMI_19 & 331 & human faeces & NZ_NOUW00000000.1 & 63 & 2.88 & 63 & [34] \\
\hline
\end{tabular}

${ }^{\mathrm{a}}$ Strains with completed circular genomes are highlighted in bold

birect data submission to NCBI Genbank

existing phylogenetic lineages within the species, we performed phylogenetic analysis of amino acid sequences of a set of the most evolutionary conserved proteins within the family Ruminococcaceae. An OrthoMCL [43] clustering was performed where $30 \mathrm{~F}$. prausnitzii genomes and an additional 23 genomes of type strains representing 23 species of the family Ruminococcaceae were included (including the genome of the Gemmiger/Subdoligranulum sp. strain APC924/74 sequenced within this study, Additional file 3: Table S1).

This family-wide analysis identified 13,261 orthologous groups of protein-coding genes and 21,324 singletons.
Hierarchical clustering analysis based on a presence/absence matrix clearly separated $F$. prausnitzii strains from the rest of the family members, while placing the Gemmiger/Subdoligranulum group of strains in close vicinity (Fig. 5a). This suggests that some features exist in the gene complements of $F$. prausnitzii and a related highly predominant gut symbiont Gemmiger/Subdoligranulum [40], which make them distinctively different from the rest of the family. Of the identified 13,261 orthologous gene groups only 245 were conserved across the family; non-paralogous, single-copy house-keeping genes suitable for phylogenetic inference (Additional file 4: Table 


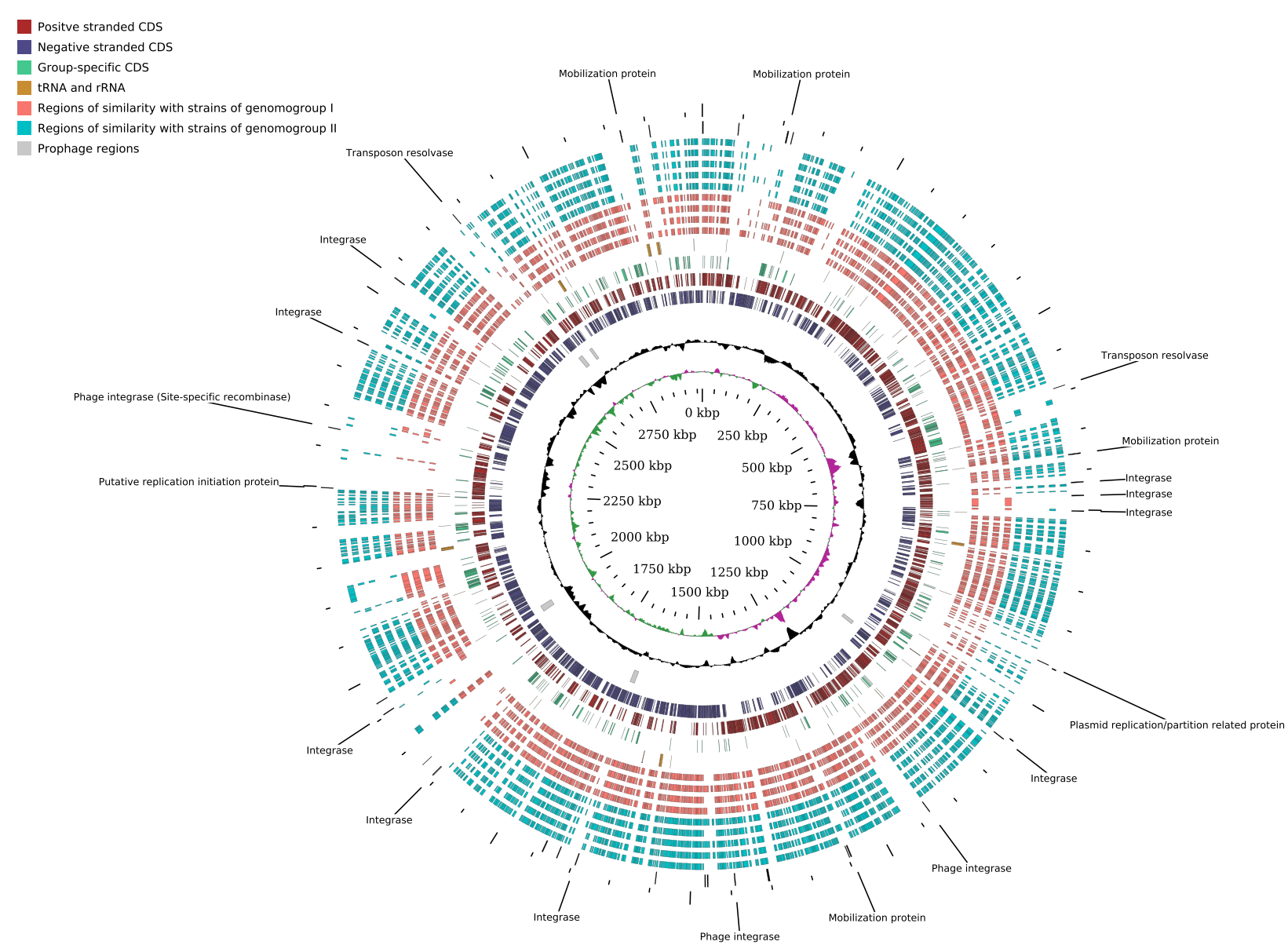

Fig. 1 Circular map of complete 2.97 Mbp chromosome of F. prausnitzii APC918/95b, representative for phylogroup I. Innermost circle (green and purple), GC skew; circle 2 (black), G + C content; circle 3 (grey), predicted prophage remnants; circles 4 and 5 (dark red and blue), ORFs located on + and - DNA strands respectively; circle 6 (green), genes specific for genomogroup l; circle 7 (orange), tRNA and rRNA genes; circles 8-11 (pink), homologous genomic segments of $>1000$ nt from other representatives of genomogroup I (APC924/119, APC923/51-1, ATCC 27768 ${ }^{\top}$, CNCM 4573,); circles 12-16 (light blue), homologous genomic segments of > 1000 nt (aligned by Mauve) from representatives of genomogroup II (APC942/30-2, A2-165, APC922/41-1, APC923/61-1, KLE1255); circle 17, genes annotated as integrase, recombinase, replication initiator protein, mobilization protein, transposase

S2). A phylogenetic tree was constructed based on multiple alignment of concatenated sequences of the 245 house-keeping proteins, which characterizes $F$. prausnit$z i i$ as a monophyletic group of strains, producing a common deep branch within the phylogenetic structure of the family Ruminococcaceae (Fig. 5b). The closest relatives to $F$. prausnitzii taxa were the human gut symbionts Gemmiger/Subdoligranulum and Ruthenibacterium lactatiformans [44].

Importantly, the $F$. prausnitzii branch itself produced a clear and statistically significant split into three species/subspecies level groups which partly coincided with the phylogroup division based on the $16 \mathrm{~S}$ rRNA gene sequences [31], and the ANI clusters seen in our analysis (Fig. 4). For consistency with previously used taxonomy these newly observed phylogroups were named as I (corresponding to $16 \mathrm{~S}$
rRNA phylogroup I), IIa and IIb (together corresponding to $16 \mathrm{~S}$ rRNA phylogroup II). It should be noted however that phylogenetically, phylogroup IIa, containing strains KLE1255, CNCM 4540, CNCM 4541 CNCM 4542, CNCM 4544, seems to have a common ancestor with phylogroup I, not IIb (four out of these five strains formed a separate cluster by ANI as shown in Fig. 4). This implies that the ANI and $16 \mathrm{~S}$ rRNA-based phylogroup II has a heterophyletic origin, despite clear commonalities of gene complement between its different member strains.

Interestingly, several phylogroup II strains with distinctly different genotypes (APC942/8-14-2, APC942/ 30-2 and APC942/32-1) were isolated from a single donor, highlighting that multiple related but distinct strains of $F$. prausnitzii can simultaneously be present in the gut microbiome of an individual. 


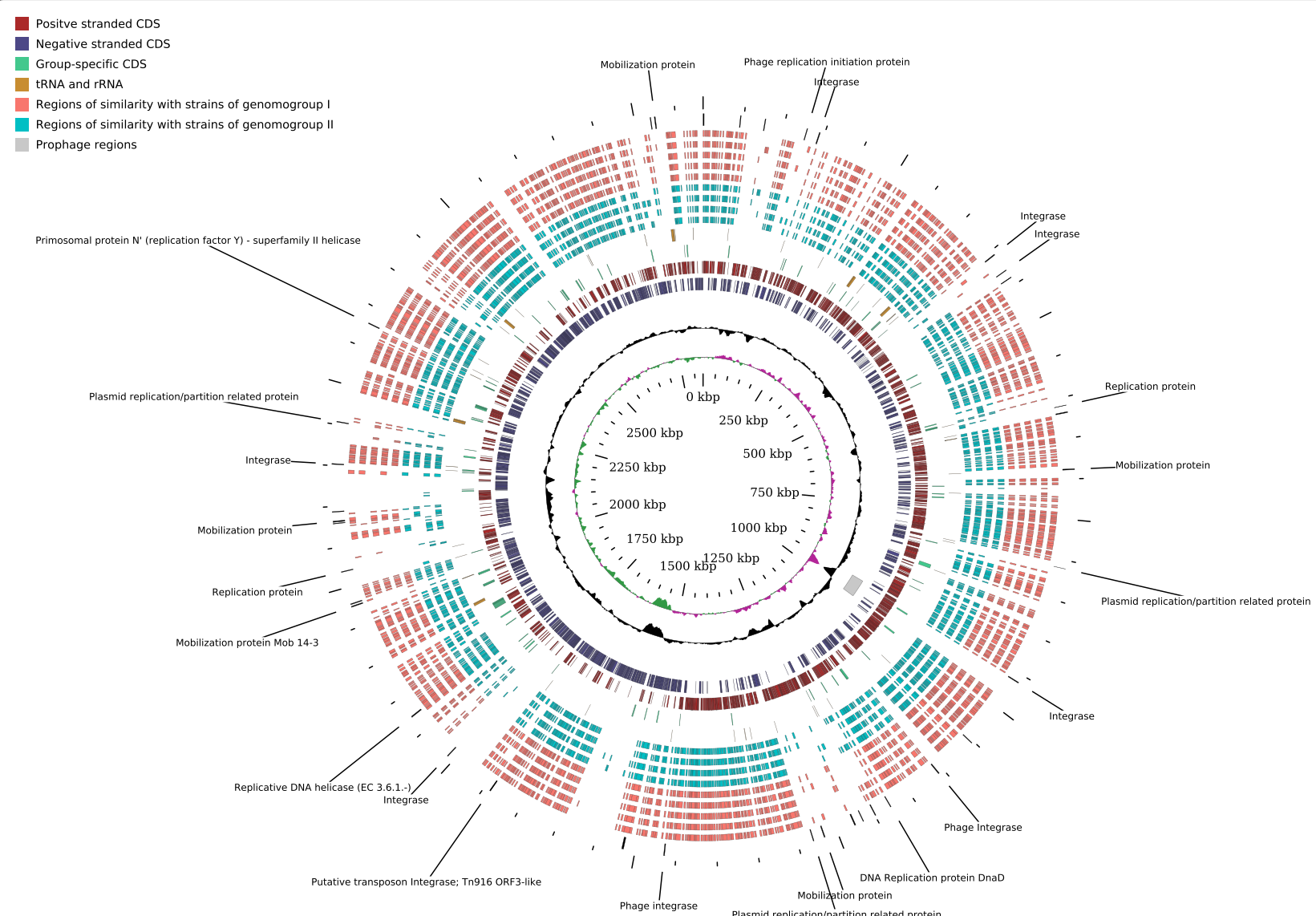

Fig. 2 Circular map of complete 2.83 Mbp chromosome of F. prausnitzii APC942/30-2, representative for phylogroup II. Innermost circle (green and purple), GC skew; circle 2 (black), G + C content; circle 3 (grey), predicted prophage remnants; circles 4 and 5 (dark red and blue), ORFs located on + and - DNA strands respectively; circle 6 (green), genes specific for genomogroup I; circle 7 (orange), tRNA and rRNA genes; circles 8-11 (light blue), homologous genomic segments of > 1000 nt from other representatives of genomogroup II (A2-165, APC922/41-1, APC923/ 61-1, KLE1255); circles 12-16 (pink), homologous genomic segments of > $1000 \mathrm{nt}$ (aligned by Mauve) from representatives of genomogroup I (APC918/95b, APC924/119, APC923/51-1, ATCC 27768 ${ }^{\top}$, CNCM 4573); circle 17, genes annotated as integrase, recombinase, replication initiator protein, mobilization protein, transposase

Protein-coding capacity and functional specialization of $F$. prausnitzii genomes

To obtain an insight into the structure of the core- and pan-genomes of F. prausnitzii, as well as to confirm the existence of two or more species/subspecies level groups within $F$. prausnitzii with distinct functional properties, we performed de novo sequence clustering using the OrthoMCL pipeline of gene products encoded by 31 complete and partial $F$. prausnitzii genomes with subsequent annotation of consensus protein sequences using the COG database (Additional file 5: Figure S3, Additional file 6: Table S3).

This analysis demonstrated the presence of 6619 protein orthologs and 4011 singletons. For 806 protein coding genes, multiple paralogs or duplicated orthologs were present in the same bacterial strain. Some extreme examples of multiple members per genome of the same orthologous group included; group $\mathrm{fp} \_1$, consisting of TraG conjugal transfer proteins (2-14 members per genome); group fp_4 of TraE conjugal transfer proteins (1-11 representatives per genome), as well as groups $\mathrm{fp} \_2$, fp_3, fp_5, fp_7 and fp_9, each of which was represented by up to 8 copies per genome. Those latter five groups represented site-specific recombinases (fp_2, $\mathrm{fp} \_5$ ), type Ia DNA topoisomerases (fp_3) and DNA relaxase/mobilization nuclease (fp_7, fp_9). Interestingly, none of the most prevalent orthologous groups represented DNA transposases.

The core genome of $F$. prausnitzii is composed of 1333 protein-coding gene orthogroups including 1245 orthogroups with a single member per genome. Analysis of core- and pan-genome accumulation curves suggests that a sample of $31 \mathrm{~F}$. prausnitzii genomes was sufficient to define the core-genome (Additional file 7: Figure S4a). By contrast, the pangenome accumulation curve continues to increase even upon reaching 10,630 genes.

Due to the variable number of orthologs and paralogs representing the same orthologous cluster the actual 


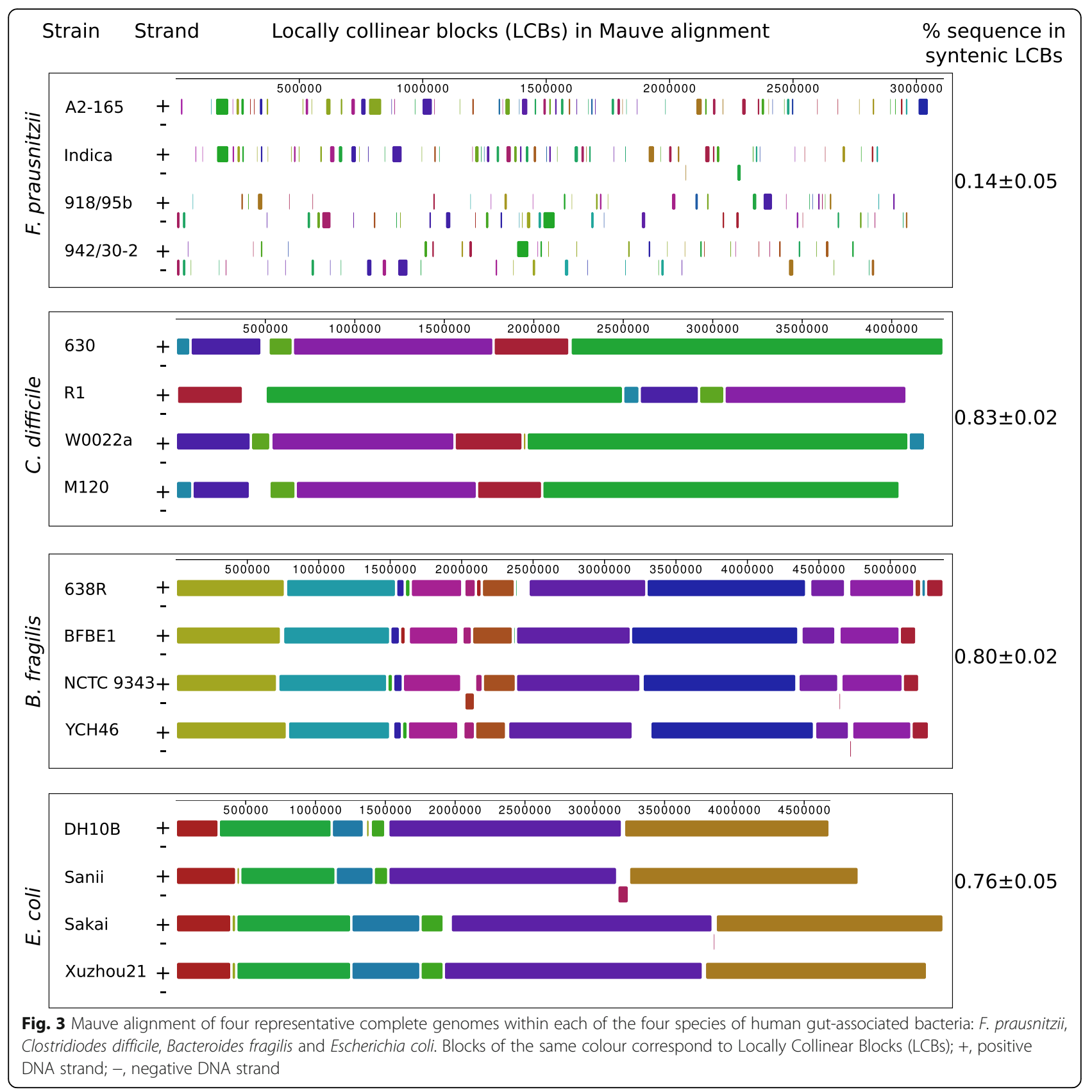

counts of predicted core genes may vary from 1361 (strain APC942/8-14-2) to 1409 (CNCM_4573), and an even greater degree of variation exists in the accessory genome, with gene counts ranging from 1139 (APC923/ 61-1) to 1826 (L2/6, Additional file 7: Figure S4b). Of 10,630 putative protein products encoded by the $F$. prausnitzii pangenome, a large number (6173) could not be positively annotated using the COG database. Of the remaining 4457 orthologous groups, the majority belonged to COG categories G (carbohydrate transport and metabolism), L (replication, recombination and repair), $\mathrm{K}$ (transcription), and $\mathrm{M}$ (cell wall/ membrane/envelope biogenesis). Interestingly, a considerable number of orthologs also belong to categories $\mathrm{V}$ (defense mechanisms, 274 orthologs) and X (mobilome: prophages, transposons, 151 orthologs; Additional file 7: Figure S4c). These latter groups included, among others, 25 separate orthologous groups of core genes coding for predicted $\mathrm{Na}^{+}$-driven and $\mathrm{ABC}$-type multidrug resistance pumps, as well as a phage terminase large subunit (fp_117) present in the core genome.

An exploratory comparative analysis of genomes of various $F$. prausnitzii strains based on shared orthologous gene groups revealed a clear separation of two 


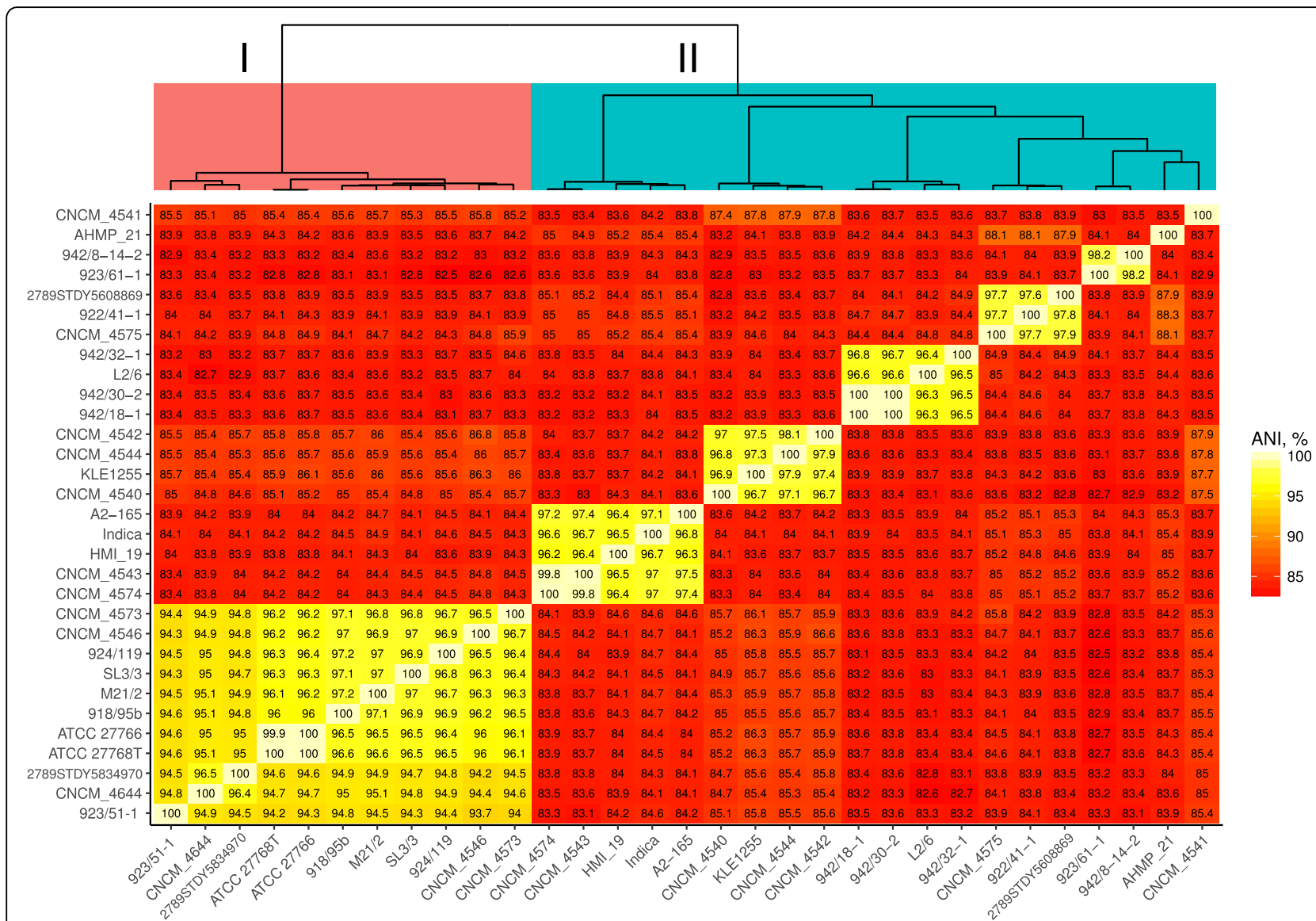

Fig. 4 BLASTn-based average nucleotide identity (ANIb) between available 31 complete and draft genomes of F. prausnitzii. Dendrogram on top built by hierarchical clustering using Ward.D2 algorithm

genomogroups (GAP statistic predicts two as the optimum number of clusters) along PCA axis 1 , which explained $14 \%$ of the variation observed in the dataset (Fig. 6a). This separation into two genomogroups agrees with two phylogroups identified by $16 \mathrm{~S}$ rRNA gene sequencing in [31], but contradicts our phylogenetic model based on conserved proteins, which placed some of the 16S rRNA phylogroup II strains (group IIb mentioned above) onto a branch common with phylogroup I. This is also in contradiction to findings of three genomogroups in a study where a combination of $16 \mathrm{~S}$ rRNA gene analysis, ANI, and wgMLST approaches were used [34].

Having defined those two clear functional genomogroups (as opposed to evolutionary phylogroups discussed above), we determined which particular groups of genes drove the separation. As expected, it is mainly the components of the accessory genome that are responsible for the split between genomogroups. Of the 22 COG categories identified in the $F$. prausnitzii pangenome, only six (K, transcription; $\mathrm{T}$, signal transduction; $\mathrm{V}$, defense mechanisms; $\mathrm{X}$, mobilome: prophages, transposons; and $\mathrm{Q}$, secondary metabolites biosynthesis; $\mathrm{N}$, cell motility) were over-represented in the accessory genome ( $p<0.05$ in Wilcoxon test with Bonferroni correction), which makes them potentially responsible for functional specialization of the two genomogroups (Fig. 6b). Upon closer examination, 468 orthologous groups of gene products were differentially distributed between the two genomogroups $(p<0.05$ in Wilcoxon test with FDR correction; Additional file 8: Figure S5, Additional file 9: Table S4) with only 237 having functionally characterized homologs from the COG database (Fig. 7). Of the latter, 36 belonged to COG category G (carbohydrate transport and metabolism), another 36 were connected with transcription (category $\mathrm{K}$ ), 31 were involved in amino acid transport and metabolism (E), 21 in cell wall/membrane biogenesis (category M), 19, 17 and 10 were linked to signal transduction $(\mathrm{T})$, defense mechanisms $(\mathrm{V})$ and energy production and conversion $(C)$, respectively.

In total, 381 orthologous groups defined genomogroup I, while genomogroup II was characterized by the presence of only 93 specific orthologous groups (Fig. 7, Additional file 8: Figure S5). Orthologues specific to genomogroup I mainly belong to COG categories G (carbohydrate transport and metabolism) and K (transcription), 


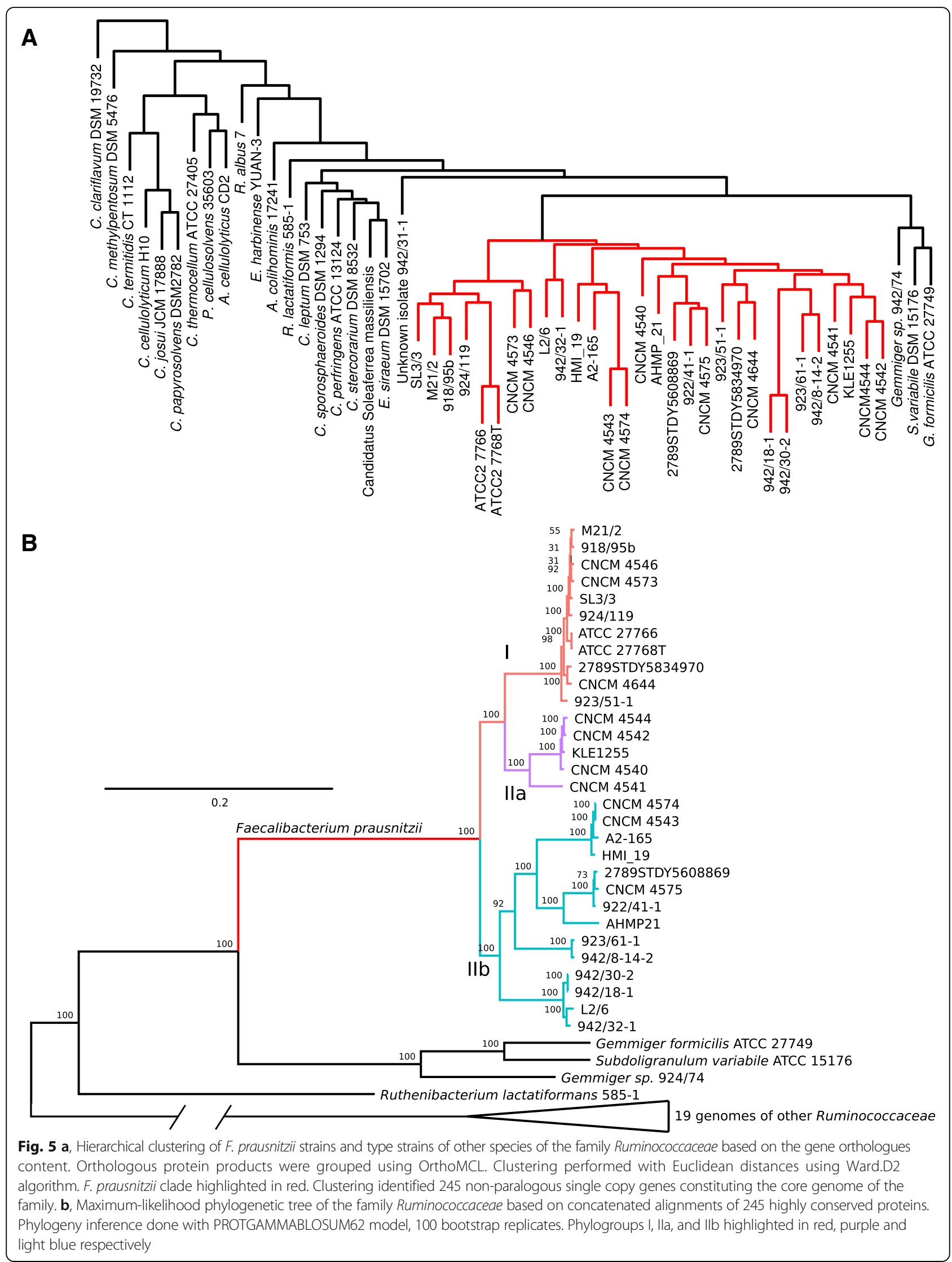




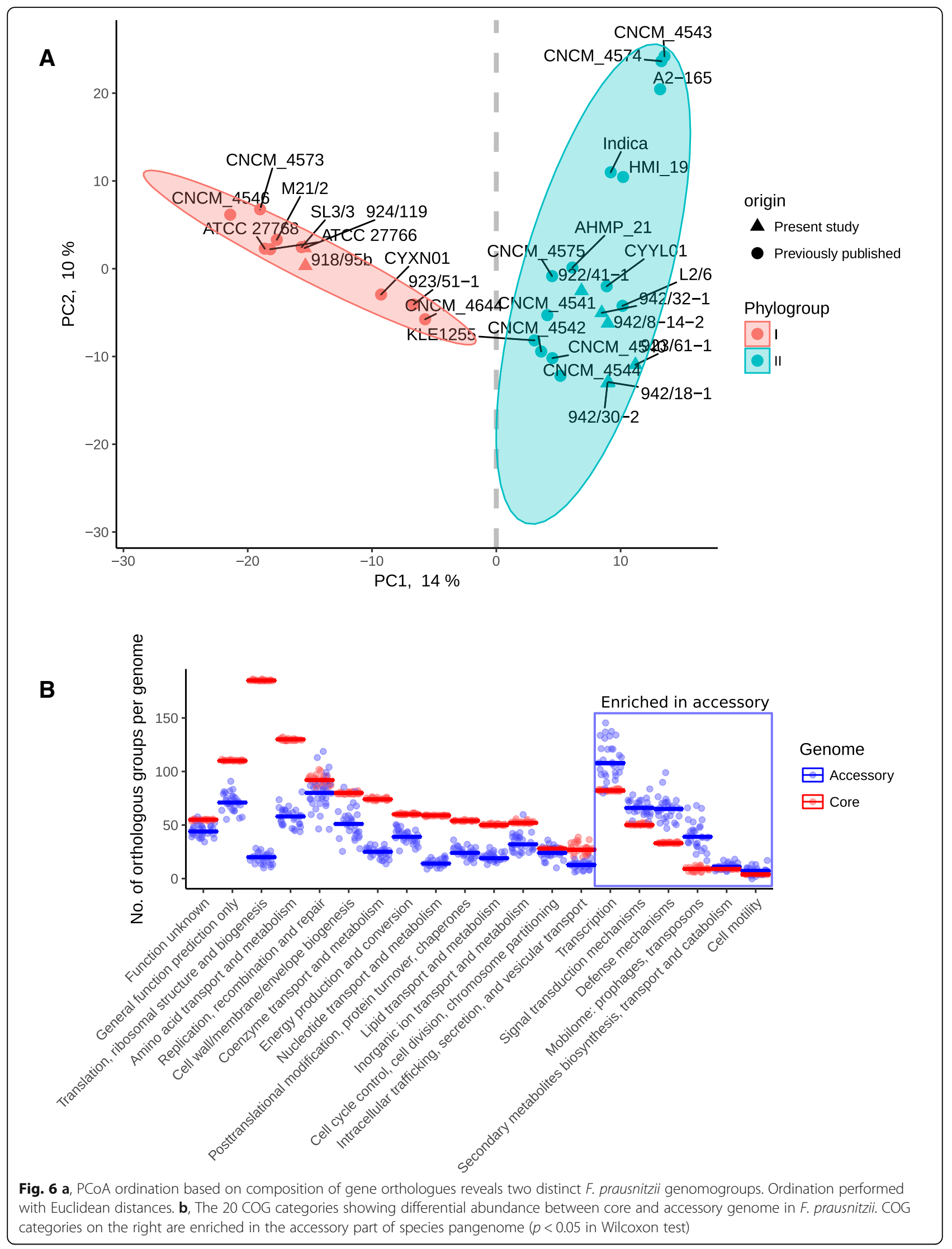




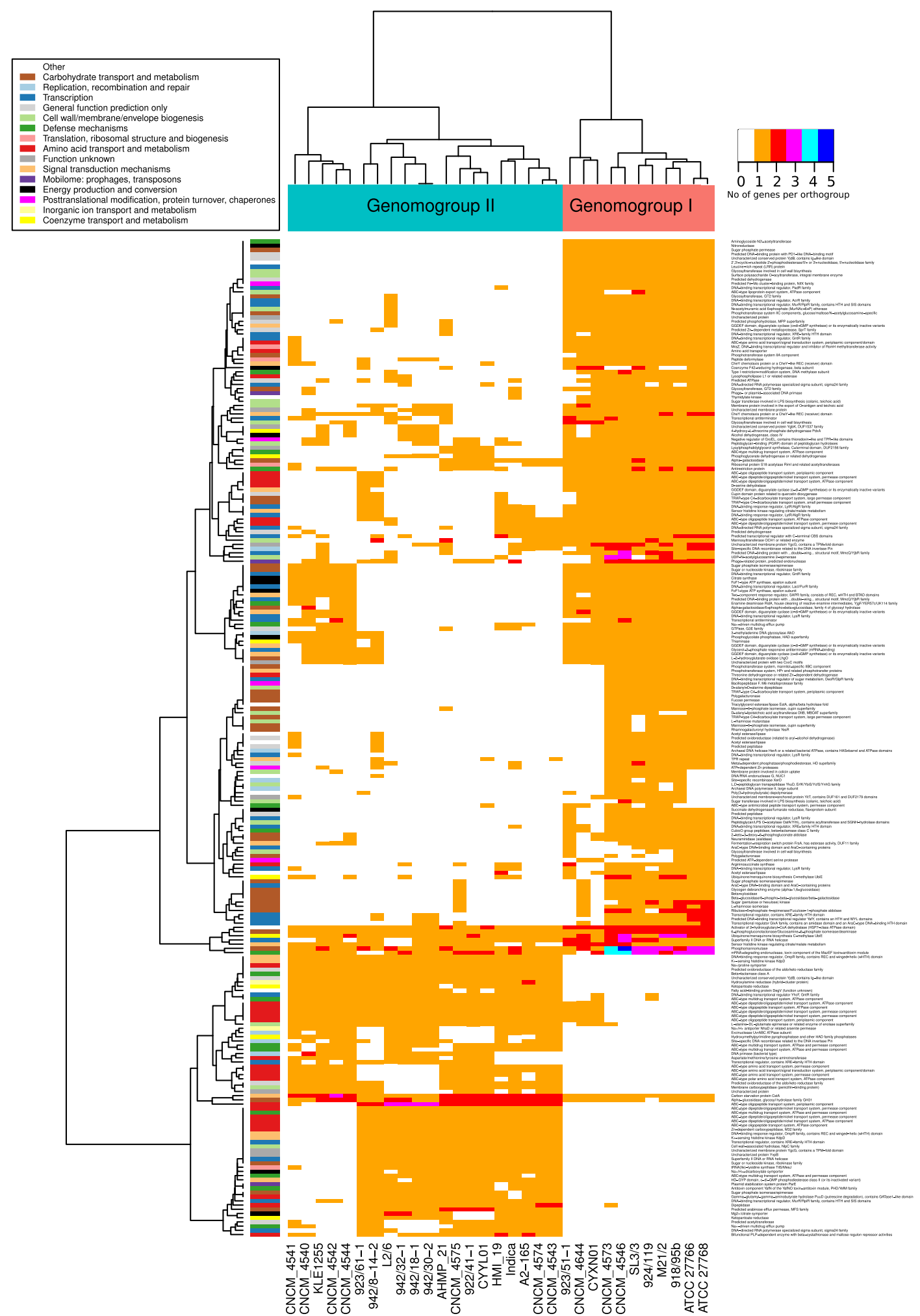

Fig. 7 A heatmap of gene orthologues differentially abundant between the two F. prausnitzii genomogroups ( $p<0.05$ in Wilcoxon test). Dendrogram on top reflect hierarchical clustering using Ward.D2 algorithm. Only groups with positive COG annotation are shown, see annotation bar on the left and the relevant legend inset. Orange colour in heatmap corresponds to single copy orthologues, other colours used for orthologous groups with multiple member per genome (see colour code on the right). An expanded version of this heatmap is given in Additional file 8: Figure S5, where all orthologous groups are shown, regardless of COG annotation availability 
$\mathrm{M}$ (cell wall/membrane biogenesis), and $\mathrm{T}$ (signal transduction) whereas genomogroup II-defining orthologues mainly belong to category $\mathrm{E}$ (amino acid transport and metabolism), $\mathrm{V}$ (defense mechanisms), and $\mathrm{K}$. A number of specific enzymatic functions are associated with a particular genomogroup. For example aminoglycoside N3' - acetyltransferase, nitroreductase, neuraminidase (sialidase), polygalac turonase, fucose permease and $\beta$-xylosidase were almost exclusively found in genomogroup $\mathrm{I}$, while $\mathrm{Na}^{+}$/proline symporter, class A $\beta$-lactamase, $\gamma$ - glutamyl $-\gamma-$ aminobutyrate hydrolase, as well as a large list of putative ABC-type oligopeptide transporters were associated with genomogroup II (Fig. 7, Additional file 9: Table S4). We also noticed the presence of bile salt hydrolase (BSH) in a subgroup of four genomogroup II strains (CNCM_4543, CNCM_4547, A2-165 and HMI_19).

In order to identify whether the genomogroup-defining genes were distributed along the genomes or were grouped into operons we mapped their location onto complete circular chromosomes of strains APC918/95b (genomogroup I) and APC942/30-2 (genomogroup II). As shown in Figs. 1 and 2 (ring 6) these genes were very often grouped into operon-like gene constellations, rather than randomly distributed. The most prominent genomogroup I-specific operons include an oligopeptide $\mathrm{ABC}$ transporter operon opp $A B C D F(1,782,575-1,788,510$ in APC918/95b), a rhamnose utilization operon $(2,451$, 480-2,458,052 in APC918/95b), and an N-acetylmuramic acid utilization operon juxtaposed with a putative operon containing a sialidase gene $(2,838,329-2,853,903$ in APC 918/95b). Similarly, several genomogroup II-specific operons could be identified, including two distinct oppABCDF oligopeptide ABC transporters (1,885,234-1, 895,957 and 2,274,107-2,280,346 in APC942/30-2).

Distribution of carbohydrate active enzyme demonstrate clear separation between the two genomogroups (Additional file 10: Table S5). The key genes required for utilization of neuraminic acid ( $\mathrm{N}$-acetylneuraminate lyase, fp_255; N-acetylmannosamine kinase, fp_304; Nacetylmannosamine-6-phosphate 2-epimerase, fp_257) are present in all strains. However, the putative sialidase (fp_2493, GH33 family) present only geonomogroup I could be a useful trait for phylogroups delineation. Agreeing with that, genomogroup I representative strains (APC918/95b and APC924/119), but not genomogroup II strains demonstrated growth on $\mathrm{N}$-acetylneuraminic acid.

Four genomes belonging to genomogroup I (M21/2, APC918/95b, 2789STDY5834970 and SL3/3) harbour a large cluster $(2,076,504-2,140,551$ in APC918/95b) of carbohydrate active genes including $\beta$-1,4-mannanase (family GH113) and $\alpha-1,6$-mannanase (family GH76). These enzymes are absent in other strains. This island also contains other mannose catabolism-related enzymes (phosphomannomutase, mannose-6-phosphate isomerase).
Despite carrying these genes, APC918/95b was unable to grow on $\alpha$-mannan. Family GH32 includes various saccharases and inulinases and was found to be present in most of the strains, with exception of M21/2 and SL3/3. In our hands, strains APC918/95b and APC924/119, but not APC942/30-2 and A2-165 were capable of growth on inulin, despite all of them possessing GH32. In agreement with earlier reports, all of the strains were able to grow on D-cellobiose, D-galacturonic acid and D-maltose [31].

\section{F. praunitzii mobilome and prophage content}

We were unable to identify circular plasmids in $F$. prausnitzii. However, in the related strain Gemmiger/Subdoligranulum sp. APC924/74 sequenced as part of this work, two circular plasmids were readily identifiable; a large low-copy number pAPC924_74_29.8 kb (coverage ratio to chromosomal DNA 1.75:1) and a smaller cryptic high copy number pAPC924_74_1.9 kb (coverage ratio to chromosomal DNA 65.6:1).

We also investigated newly and previously sequenced F. prausnitzii genomes for the presence of prophages. A total of 89 prophage and prophage-like elements were identified in the genomes of $31 \mathrm{~F}$. prausnitzii strains (1-6 elements per strain), with sizes ranging from 6.3 to $64.6 \mathrm{~kb}$. These putative prophage regions contained 7-78 protein coding genes, of which 1-26 could be annotated using COG database and 3-33 could be annotated using phage-specific pVOG database.

Of the 89 prophage regions only 22 (median length 36 $\mathrm{kb}$ ) were predicted as being complete or almost complete with high level of confidence (PHASTER prediction confirmed with Virsorter algorithm). Of these, six belong to the viral family Myoviridae and 15 to the Siphoviridae. They contained a median number of 47.5 genes per prophage, with a median count of 19 being identifiable using the pVOG database (Additional file 11: Table S6). Hierarchical clustering of high-confidence prophage regions based on the percentage of shared orthologous genes with a cut-off level set at $40 \%$, enabled us to identify 6 clusters with two-four members per cluster and six orphan sequences. Analysis of presence of different prophage classes across the 31 genomes shows that only 15 contained one to three prophages and little or no correlation could be seen between prophage content and membership of a strain in one of the two F. prausnitzii phylogroups/genomogroups.

Of the 22 prophages predicted to be complete or almost complete, 11 were already described earlier [45]. Of the remaining 11 novel prophages, three were from strains isolated in this study and eight were from previously available genomes. Twenty of the 22 prophages fit into the various genera described by Cornuault et al. 
[45] while only two of the novel prophage separate into a new genus-level group.

In order to assess functionality of prophages as well as to discover potential novel prophage sequences we performed a prophage induction experiment on eight $F$. prausnitzii strains (A2-165, APC942/30-2, APC922/411, APC923/51-1, APC923/61-1, APC942/8-14-2, ATCC $27768^{\mathrm{T}}$, ATCC 27766), as well as the Subdoligranulum/ Gemmiger sp. strain APC924/74. Metagenomic sequencing of DNA extracted from concentrated viral fractions from the supernatant was used to detect virus like particle (VLP) associated prophage DNA. Contrary to our expectations, DNA of only one F. prausnitzii prophage, A2165_phage_2corr (termed MushuA2-165 in Cornuault et al. study), was present in VLPs at levels exceeding those of background contamination with bacterial genomic DNA. Also, we observed induction of a $\sim 40 \mathrm{~kb}$ prophage (APC924/74_phage_1) from Subdoligranulum/Gemmiger sp. APC924/74. Quantitative PCR was also used to confirm that induction of A2-165_phage_2corr happened spontaneously as well as in response to mitomycin $\mathrm{C}$ treatment, with levels of spontaneous induction only marginally lower than those achieved with mitomycin $C$ (Additional file 12: Figure S6). When culture supernatants from A2-165 and APC924/74 were tested individually in plaque-formation assays against the same panel of eight strains no plaques could be seen. This suggests that induced prophages were either not fully functional or had extremely narrow host ranges. In fact, the hypothetical protein encompassing the A2-165_phage_2corr attB sequence belongs to an orphan orthologous group fp_9714 represented by a single member only found in strain A2-165.

\section{Discussion}

F. prausnitzii is one of the most abundant species of bacteria found in the human gut [4-6, 8]. In recent years it has been the subject of a significant volume of research, due to its abundance, its potential link to IBD and the drive to create 'next generation probiotics' [33]. It was established early on that $F$. prausnitzii can be split into two phylogroups based on the sequence of the $16 \mathrm{~S}$ rRNA gene, and that each has slightly different biochemical characteristics and response to inflammation in the human gut [31,35]. Apart from 16S rRNA gene comparisons and ANI analysis [34] of draft genome sequences, the understanding of the genetic differences between the two phylogroups has not been fully explored. In this study we de novo sequenced genomes of 11 strains of $F$. prausnitzii, including two where we generated complete circular chromosomes, and compared them with a further 20 high quality draft and completed genomes available from public databases.
Using a combination of ANI analysis, reconstruction of the phylogeny of 245 essential house-keeping genes conserved at the level of the family Ruminococcaceae and genome wide analysis of protein coding gene complement, we attempted to interrogate the separation of F. prausnitzii into two phylogroups and to identify functional genetic traits associated with these subgroups. We observed a number of discrepancies between the methods. Conserved protein analysis (phylogroups) and genome wide gene complement analysis (genomogroups) identified two different clusters of strains, with only a partial overlap (Figs. 5b and 6a). Such discrepancies are not entirely unexpected, given the large number of putative ICE/IME in F. prausnitzii genomes which can be responsible for extensive acquisition of genes originating from related and unrelated bacterial species. One can hypothesize therefore, that rapid horizontal gene transfer (HGT) events may be the main driving force shaping the composition of individual $F$. prausnitzii genomes and that the current gene complement may not accurately reflect the long term evolutionary history of each strain [46].

When looking at the average nucleotide identity of different $F$. prausnitzii strains it was obvious that there was a very low level of ANI between genomogroups, and even between some members of genomogroup 2. The level of intraspecies ANI of $F$. prausnitzii is significantly lower than the $95 \%$ threshold currently suggested as defining a bacterial species [41, 42], while an even stricter cut-off level of $96.5 \%$ has been suggested recently [47]. The ANI analysis also confirmed previous observations regarding genomogroup I and independently grouped all relevant strains together both at the level of reciprocal identity between the genomes (Fig. 4) and at the level of reciprocal sequence coverage (Additional file 2: Figure S2). However, a coherent genomogroup II doesn't seem to exist when ANI is considered. Instead, strains forming genomogroup II separated into five smaller clusters and two solitary genomes. This observation, together with the low level of genome synteny suggests that multiple diverse phylogenetic lineages exist within the species of $F$. prausnitzii. Moreover, separation of $F$. prausnitzii into several species or subspecies level taxa may be needed to more accurately reflect the evolutionary and functional separation of strains.

It was of particular interest to look into the functional difference between the two genomogroups, given their differential response to the intestinal inflammation [35]. Our analysis indicated that 381 orthologous groups defined genomogroup I, while 93 orthologous groups defined genomogroup II. The most differential functional subsets of genes were those involved in catabolism of carbohydrates and amino acids, highlighting the difference in nutritional strategies between the two genomogroups. The exclusive 
ability of genomogroup I strains, which possess a predicted sialidase gene (orthologous group fp_2493), to grow on $\mathrm{N}$-acetylneuraminic acid further supports these observations. It is also interesting that the COG category $\mathrm{V}$ (defense mechanisms - mostly multidrug efflux pumps) were overrepresented in genomogroup II strains, which in turn were shown to be more abundant in patients suffering from $C D$ [48].

Our analysis revealed a striking lack of synteny between the four complete circular genomes of $F$. prausnitzii, representing both genomogroups (Fig. 3, Additional file 1: Figure S1). This shows that even when two members of the same genomogroup are compared to each other the levels of synteny observed are very low. To put this into perspective we demonstrated the significantly higher synteny levels in four complete genomes of four strains of three other genera representing diverse bacterial clades colonizing the human gut; $C$. difficile, B. fragilis and $E$. coli. We note that in a number of cases the regions of low synteny are flanked by genes coding for integrases, site-specific recombinases, plasmid replication and mobilization proteins. The size of LCB blocks is also worth noting as it shows that only small portions of the genomes are conserved when shuffling occurs. These findings are consistent with our observations of large numbers of mobile genetic elements distributed around the $F$. prausnitzii genomes. Interestingly, while $F$. prausnitzii genomes harbor considerable numbers of integrative and conjugative (mobilizable) DNA elements (ICE/IME, [49]), they lack complete prophages, classical transposons, IS and other transposable elements. One could speculate that the large numbers of integrative elements might be responsible for frequent HGT and intragenomic recombination events leading to the low levels of both intraspecies genomic similarity and synteny seen in $F$. prausnitzii. Genome shuffling might be advantageous in that it could lead to rapid phenotypic improvement in bacteria [50]. The exact mechanisms underlying this genome reshuffling and the significance of this phenomenon to the adaptation of $F$. prausnitzii to the human gut environment, as well as possible consequences to human health, remain to be elucidated.

Our analysis suggests that there are large differences between the strains of $F$. prausnitzii on a genomic level, and that current unification of all of these strains into a single species is not reflective of their phylogeny and functional diversity. Based on the combination of ANI of genomic sequences, phylogenetic analysis of core proteomes and functional differences we propose to separate the species Faecalibacterium prausnitzii (Duncan et al., 2002 [3]) into two new species level taxa. While members of phylogroup I strains fulfil the usual genomic criteria for a separate species - a monophyletic group of strains with $\mathrm{ANI} \geq 95 \%$ [41, 42], phylogroup II presents a much more loosely connected group of strains from a phylogenetic point of view. Phylogroup II strains do not share sufficient level of nucleotide identity with either phylogroup I strains or between themselves (Fig. 4). This group of strains is paraphyletic when core proteome-based phylogeny is considered and demonstrates considerable heterogeneity of phenotypes [31]. At the same time these strains form several clusters of high ANI, while leaving two strains as singletons (Fig. 4). As one of the most abundant bacteria in the human gut, as well as being common in the microbiota of other vertebrates $[51,52]$, it is not surprising that faecalibacteria demonstrate high levels of genomic diversity. It seems likely that as more strains belonging to the genus Faecalibacterium are isolated and more genomic, genetic and phenotypic data accumulates, it will become possible to describe several species level taxa within the genus Faecalibacterium, replacing the current single species. At the moment it seems logical to separate members of phylogroup I into a new species while leaving $F$. prausnitzii as a provisional taxon until it becomes possible to describe further new species within it. Given its common use in various studies as a model $F$. prausnitzii strain we propose the strain A2-165 [3] as the neotype strain of the amended species $F$. prausnitzii (which includes various heterogenous phylogroup II strains). We also propose to create a new species $F$. moorei to include all phylogroup I strains. The original type strain of the genus Faecalibacterium (Duncan et al. 2002 [3]) is proposed to serve as a type strain of the species under the new name Faecalibacterium moorei nom. Nov. ATCC $27768^{\mathrm{T}}$. We choose Faecalibacterium moorei (Moo.rei. M.C. gen. N. moorei of Moore; moorei) in honour of Walter Edward C. Moore (1927-1996), a prominent American bacteriologist famous for developing techniques to grow anaerobic bacteria, and one of the authors of the original description of 'Fusobacterium prausnitzii'.

\section{Emended description of Faecalibacterium prausnitzii}

Cells are Gram-stain-negative, non-motile, non-sporeforming and strictly anaerobic. Genomic DNA G + C content is $55.7-63.0 \mathrm{~mol} \%$. Genome size is $2.68-3.32$ $\mathrm{Mb}$. Strains of the species show high level of genetic heterogeneity with median ANI of just $85 \%$. Cells are able to utilize D-cellobiose, D-galacturonic acid, D-maltose as a sole carbohydrate substrate. The rest of the species characteristics are as described for Fusobacterium prausnitzii ATCC $27768^{\mathrm{T}}$ by Cato et al. (1974). The neotype strain is Faecalibacterium prausnitzii A2-165 ${ }^{\mathrm{T}}$ (=DSM

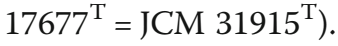

\section{Description of Faecalibacterium moorei nom. nov.}

Faecalibacterium moorei (Moo.rei. M.C. gen. N. moorei of Moore; moorei named in honour of Walter Edward C. 
Moore [1927-1996], a prominent American bacteriologist, famous for developing techniques to grow anaerobic bacteria, and one of the authors of the original description of 'Fusobacterium prausnitzii').

Cells are Gram-stain-negative, non-motile, non-sporeforming and strictly anaerobic. Genomic DNA G + C content is $54.9-56.5 .0 \mathrm{~mol} \%$. Genome size is $2.92-3.42$ $\mathrm{Mb}$. Strains of the species share 94-97\% ANI in genome sequences. Cells are able to utilize D-cellobiose, D-galacturonic acid, D-maltose, and $\mathrm{N}$-acetylneuraminic acid as a sole carbohydrate substrate. The rest of species characteristics are as described for Fusobacterium prausnitzii ATCC $27768^{\mathrm{T}}$ by Cato et al. (1974) and Duncan et al. (2002). The type strain is Faecalibacterium moorei nom. nov. ATCC $27768^{\mathrm{T}}\left(=\right.$ NCIMB $\left.13872^{\mathrm{T}}\right)$.

\section{Materials and methods}

\section{Isolation and cultivation of $F$. prausnitzii and related} bacteria

F. prausnitzii strains isolated and used in this study were grown on either M2GSC plates [53] containing 1.5\% of bacto agar, M2GSC broth clarified by filtration through a $0.45 \mu \mathrm{m}$ pore polyethersulfone (PES) membrane filter, or supplemented YCFA broth media [3]. YCFA broth was modified by supplementing with cellobiose, glucose, starch and maltose (each carbohydrate at $2 \mathrm{~g} / \mathrm{L}$ ). Both types of media were supplemented with $1 \mathrm{mg} / \mathrm{L}$ of resazurin indicator for monitoring of redox potential. All manipulations were performed in a Simplicity-888 (Plas-Labs) automatic anaerobic chamber at $37^{\circ} \mathrm{C}$ in an atmosphere of $80 \% \mathrm{~N}_{2}, 10 \% \mathrm{H}_{2}, 10 \% \mathrm{CO}_{2}$ in the presence of a palladium catalyst canister for removal of residual oxygen. Broth media were taken into an anaerobic chamber immediately after autoclaving, while agar plates were prepared aerobically and equilibrated in an anaerobic chamber for $24 \mathrm{~h}$ before use.

Faecal samples for isolation of $F$. prausnitzii strains were collected from 6 healthy adult Faecal samples were collected from consenting clinically healthy volunteers according to study protocol APC055, approved by the Cork Research Ethics Committee (CREC). Participants were randomly selected for sampling, did not report receiving antibiotics or probiotics at the time of collection and in the preceding 1 month, and were all residents of Cork, Ireland. Study participants are identified here as APC055_918 (25 y.o. female), APC055_919 (24 y.o. female), APC055_922 (30 y.o. male), APC055_923 (55 y.o. male), APC055_924 (44 y.o. female), and APC055_942 (34 y.o. male). Subject APC055_942 was sampled on two separate occasions with a 1 month interval. Faecal samples were delivered to the lab in tightly closed sterile containers and processed immediately upon delivery, within $3 \mathrm{~h}$ of voiding. Aliquots of $100 \mathrm{mg}$ of faeces were homogenized in $10 \mathrm{ml}$ of YCFA broth, then diluted serially in the same media with 10 -fold steps to $10^{-9}$. Dilutions $10^{-5}-10^{-9}$ were plated onto M2GSC plates and incubated for $48 \mathrm{~h}$ at $37^{\circ} \mathrm{C}$. Plates were examined for the presence of translucent round or irregular, slightly elevated, flat or umbonate colonies. Such colonies were purified by triple-streaking and then subjected to colony PCR using the MyTaq Red Mix kit (Bioline) with $F$. prausnitzii species specific primers (FPR-2F: 5'-GGAGGAAGAAGGTCTTCGG-3'; Fprau-645R: 5'-AATTCCGCCTACCTCTGCACT-3') [54]. The following PCR conditions were used: $95^{\circ} \mathrm{C}$ for 5 min, 30 cycles of: $95^{\circ} \mathrm{C}$ for $30 \mathrm{~s}, 60^{\circ} \mathrm{C}$ for $30 \mathrm{~s}$ and $72^{\circ} \mathrm{C}$ for $1.5 \mathrm{~min}$, followed by $72{ }^{\circ} \mathrm{C}$ for $7 \mathrm{~min}$ and held at $4{ }^{\circ} \mathrm{C}$. Isolates producing bands $\sim 248 \mathrm{bp}$ were subjected to nearly full length $16 \mathrm{~S}$ rRNA gene sequencing as described in [55] for confirmation of species identification. Finally, nine $F$. prausnitzii isolates were selected for genome sequencing (see Table 1) including four isolates from subject 942 (two related strains from time point 1 and two unrelated strains from time points 1 and 2), two isolates from subject 923 (unrelated strains) and one isolated from each of the remaining three subjects 918, 919, 924. Additionally, an isolate from subject 924 representing the F. prausnitzii-related group Subdoligranulum sp./ Gemmiger sp. as well as two other $F$. prausnitzii strains (ATCC 27766 and ATCC 27768 T; [1]) were subjected to sequencing.

Tests for ability to grow on a single carbohydrate substrate were performed in YCFA supplemented with either of $\alpha$-mannan, apple pectin (Sigma), D-cellobiose, D-galacturonic acid monohydrate, D-glucose, D-glucuronic acid, D-maltose, inulin from chicory (Sigma), $\mathrm{N}$-acetylneuraminic acid, soluble starch (Sigma), each at $2 \mathrm{~g} / \mathrm{L}$. Growth was assessed visually after 24 and $48 \mathrm{~h}$ of anaerobic incubation of $10 \mathrm{~mL}$ cultures at $37^{\circ} \mathrm{C}$.

\section{Draft and complete genome sequencing and assembly}

DNA extraction was performed using DNeasy Blood \& Tissue Kit (Qiagen) according to manufacturer's instructions (protocol for Gram-negative bacteria). Genomic DNA was quantified using a Qubit dsDNA HS Assay Kit (Invitrogen/ThermoFisher Scientific) and subjected to random shotgun library preparation using the Nextera XT DNA Library Preparation Kit (Illumina) and beadbased normalisation following the standard manufacturer's protocol. Ready-to-load libraries were sequenced using a proprietary modified protocol using $2 \times 300 \mathrm{bp}$ paired-end chemistry on an Illumina HiSeq 2500 platform (Illumina) at GATC Biotech AG, Germany. Two isolates (942/30-2 and 918/95b) were also sequenced using PacBio RS II platform at the same service provider.

The genome data for 20 additional $F$. prausnitzii strains were retrieved from the NCBI nucleotide database, 
including 2 complete genomes sequenced using either Pacific Biosciences PacBio RS II (strain A2-165, [34]) or a combination of Illumina HiSeq 2500 and Oxford Nanopore Technologies MinION DNA sequencing platforms (strain Indica [56]).

The quality of the raw reads was checked with FastQC v. 0.11.3. Nextera adapter removal, read trimming and filtering were performed using Trimmomatic v. 0.36 [57] in a sliding window mode (window size 4) to ensure a minimum length of 60 and minimum Phred score of 20. Reads were then assembled on a per sample basis with SPAdes v. 3.10.0 [58] in 'careful' mode using the k values of 21,33, 55 and 77. Where available, PacBio RS II subreads in '.fastq' format were added using the '-pacbio' option.

For isolates 942/30-2 and 918/95b hybrid assemblies with PacBio RS II subreads allowed for the reduction of contig counts 2 and 8 initially (N50 $=1,788,640$ and $1,783,978$, respectively). To complete the assemblies, PCR primers were designed to amplify gap regions between matched contig ends followed by Sanger sequencing of the obtained PCR products. Correct assembly was further verified by aligning quality filtered Illumina HiSeq 2500 reads back to the chromosomes using Bowtie2 v. 2.1.0 ('end-to-end' alignment mode) followed by manual inspection of alignments at the contig boundary regions visualized with Tablet v.1.17.08.17.

\section{Genome sequence annotation and analysis}

All genomes were subjected to automated functional annotation using the RAST server [59]. Classic RAST v. 2.0 along with FIGfam (release 70) were used to annotate all strains of $F$. prausnitzii. Errors and frameshifts were fixed automatically. Gaps were backfilled and metabolic models were created. Debug was not turned on and replication was disabled.

Complete genome assemblies were visualized using GView v1.7. Average nucleotide identity (ANI) analysis was performed using the whole-genome sequences employing the Python script 'pyani' [60]. The progressiveMauve command from the Mauve package [61] was used with default parameters to perform sequence alignment comparisons and to evaluate gene synteny among the genomes of $F$. prausnitzii. Synteny scores were calculated by pairwise comparison of all possible combinations of two genomes (of lengths $\mathrm{L}_{0}$ and $\mathrm{L}_{1}$, respectively) extracted from multiple genome alignment performed using Mauve. Homologous regions were taken from a '\#\#\#.backbone' file included into standard Mauve output. Mauve alignments were filtered to only include blocks of sequence $l>1000$ bp (\# of blocks in Genome $0=n$; order of homologous blocks in Genome 1: $i_{1} \ldots i_{k}$ ). Blocks of sequence appearing in the same consecutive order in a given pair of genomes (block offset $\left|i_{k}-i_{k-1}\right|=1$ ) were termed as being syntenic. Overall synteny score were calculated using the script 'synteny_v5.R' (see Additional file 1: Figure S1, Additional file 2: Figure S2, Additional file 3: Table S1, Additional file 4: Table S2, Additional file 5: Figure S3, Additional file 6: Table S3, Additional file 7: Figure S4, Additional file 8: Figure S5, Additional file 9: Table S4, Additional file 10: Table S5, Additional file 11: Table S6, Additional file 12: Figure S6) according to the following formula:

$$
\text { Synteny }=\frac{\sum_{i=1}^{n} l_{i}}{L_{0}} \times 100 \% \quad \text { where }: i \in\left\{\left|i_{k}-i_{k-1}\right|=1\right\}
$$

Orthologous gene groups in $F$. prausnitzii genomes were identified using OrthoMCL v. 2.0 [43]. Briefly, RAST annotated genes were translated to amino acid sequences and compared using a reciprocal BLASTP search (blastall v. 2.2.26 with a minimum $E$ value limit of $1 E-5$; [62]). An OrthoMCL pipeline was then used to cluster proteins into orthologous groups with an MCL inflation index of 1.5. Both protein clusters and singletons were included into the final output. Multiple alignment of members within each cluster was performed using MUSCLE v3.8.31 [63]. Consensus sequences were obtained per cluster using 'em_cons' tool from EMBOSS package v. 6.6.0.0. Consensus protein sequences were then functionally annotated using a BLAST search against the COG database (release of September, 2016; [64]). Annotation of carbohydrate active enzyme domains was performed using HMMER v3.1b1 and dbCAN database as dexribed before [65]. OrthoMCL output and COG annotation data were processed using custom built Python scripts and imported into R v. 3.2.3 for statistical analysis.

A separate OrthoMCL run that included 53 genomes (30 assemblies of $F$. prausnitzii and 23 assemblies of other member of the family Ruminococcaceae and the order Clostridiales) was performed in order to identify most conserved genes for phylogenetic inference in the broader context of the family. A set of 245 orthologous groups present in a single copy in each genome not having any paralogs was identified. Amino acid sequences within each group were aligned using MUSCLE [63] and then the alignments were concatenated. Phylogenetic inference was performed using RaxML 8.2.11 (PROTGAMMABLOSUM62 model, 100 bootstrap replicates; [66]).

\section{Prophage content analysis}

The online prophage search tool PHASTER [67] was used to search for prophages within the $F$. prausnitzii genomes. This output was further filtered using Virsorter [68] and only positive regions were selected. Family-level taxonomic ranks were assigned to prophages using Demovir (https://github.com/feargalr/ 
Demovir). Additionally, prophage protein coding genes were annotated using HMM search ('hmmscan' command from HMMER v3.1b2) against prokaryotic virus orthologous groups (pVOGs) database [69]. The results were used to generate a non-redundant catalogue of putative prophage and prophage-like element sequences.

\section{Prophage induction}

For prophage induction, overnight both cultures of $9 F$. prausnitzii strains (A2-165, ATCC 27766, ATCC 27768, 942/18-1, 942/30-2, 942/32-1, 922/41-1, 923/51-1, 923/61-1) as well as Subdoligranulum/Gemmiger sp. strain 924/74 were inoculated into $10 \mathrm{ml}$ of fresh clarified M2GSC broth at 1:100 ratio and incubated anaerobically at $37^{\circ} \mathrm{C}$. Upon reaching $\mathrm{OD}_{600}$ of $\sim 0.4$, Mitomycin C (Sigma) was added to final concentration of $4 \mu \mathrm{g} / \mathrm{mL}$ and incubation was continued overnight. Similarly, non-induced cultures were inoculated and grown overnight. Cultures were then centrifuged at $5000 \mathrm{rpm}$ for $15 \mathrm{~min}$ and supernatants were filtered through $0.45 \mu \mathrm{m}$ pore PES membrane filters. Supernatants from all Mitomycin $\mathrm{C}$ induction samples were pooled together. The same was done for control, non-induced samples. Phage particles from pooled samples were precipitated and nucleic acids were extracted using the faecal VLP nucleic acid extraction protocol described elsewhere [70].

Shotgun DNA libraries were prepared using the Nextera XT DNA Library Preparation Kit, sequenced and quality filtered as described above (see subsect. Genome sequence annotation and analysis). Reads were then assembled into contigs using SPAdes v. 3.10 .0 in 'meta' mode with the rest of the parameters set to default. Quality filtered and trimmed reads from were then aligned back do the assembled contigs using Bowtie2 v. 2.1.0 in the 'end-to-end' alignment mode [71]. A count table was generated with Samtools v. 0.1.19 which was then imported into R v. 3.2.3 for statistical analysis.

Quantitative PCR (qPCR) for detection of induced prophages was performed after extracting VLP DNA from individual culture supernatants as described before [70]. The following primers were used for amplification of fragments of A2-165_phage_1 (Lagaffe), A2-165_ phage_2corr (MushuA2-165), A2-165_incomplete_phage, F. prausnitzii 16S rRNA gene: P1-ORF1563-F1 (CCCCTACATCCGCTTCGACT), P1-ORF1563-R1 (CT CATCACCTTCCTCCGGCT), P2-ORF1593-F1 (CCCA CGCCCACATCCTTCTT), P2-ORF1593-R1 (CGGCGT ACTTCTGGTCGTT), P3-ORF3792-F1 (CCTCAGCCC CTCCCTTCAAT), P3-ORF3792-R1 (TTCGGCTTCCT CGGTTCCTT), FPR-2F, Fprau-645R (see sequence above) at final concentration of $0.2 \mu \mathrm{M}$. qPCR reactions were set up using LightCycler 480 SYBR Green I Master Mix in a LightCycler 480 System (Roche). The following
PCR conditions were used: $95^{\circ} \mathrm{C}$ for $5 \mathrm{~min}, 40$ cycles of: $95^{\circ} \mathrm{C}$ for $20 \mathrm{~s}, 60^{\circ} \mathrm{C}$ for $20 \mathrm{~s}$ and $72{ }^{\circ} \mathrm{C}$ for $20 \mathrm{~s}$. Experiment was performed with 2 independent repeats, each time using 3 biological replicates for each condition.

Additionally, an attempt was made to isolate putative induced prophages using agar overlay technique. For that $4 \mathrm{ml}$ of anaerobically prepared M2GSC soft agar (0.4\% agar) kept at $\sim 45^{\circ} \mathrm{C}$ was mixed with $300 \mu \mathrm{l}$ of one of the $F$. prausnitzii host strains (see list above) and $100 \mu \mathrm{l}$ of serially diluted pooled Mitomycin C induced supernatant from 10 bacterial strains. Mixtures were overlaid on to M2GSC agar plates and incubated anaerobically $37^{\circ} \mathrm{C}$ for $24 \mathrm{~h}$. No plaque formation could be detected on any of the plates.

\section{Statistical analysis}

Separation of $F$. prausnitzii into genomogroups by orthologous gene content was visualized using principal component analysis ('prcomp' function in $\mathrm{R} \mathrm{v.} \mathrm{3.2.3).}$ Hierarchical clustering of genomes was performed using Pvclust package for $\mathrm{R}$ [72] with euclidean distances and Ward's minimum variance method. Optimal number of clusters was determined by using Gap method from NbClust package. Distribution of COG categories between core- and accessory genome, as well as between the two genomogroups was examined using Wilcoxon test. Package ggplot2 for $\mathrm{R}$ was used for plotting.

\section{Additional files}

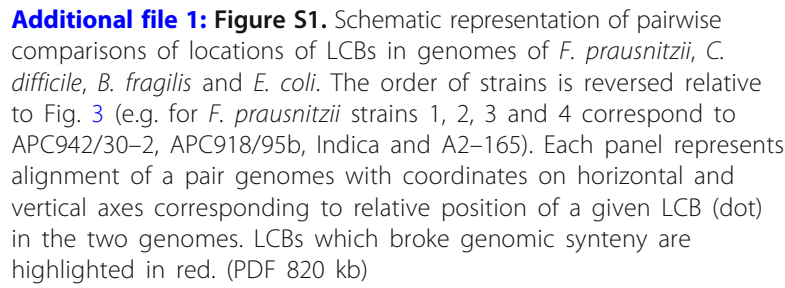

Additional file 1: Figure S1. Schematic representation of pairwise comparisons of locations of LCBs in genomes of $F$. prausnitzii, $C$. difficile, $B$. fragilis and $E$. coli. The order of strains is reversed relative to Fig. 3 (e.g. for F. prausnitzii strains 1, 2, 3 and 4 correspond to APC942/30-2, APC918/95b, Indica and A2-165). Each panel represents alignment of a pair genomes with coordinates on horizontal and vertical axes corresponding to relative position of a given LCB (dot) in the two genomes. LCBs which broke genomic synteny are highlighted in red. (PDF $820 \mathrm{~kb}$ )

Additional file 2: Figure S2. BLASTn-based pairwise sequence coverage between available 31 complete and draft genomes of F. prausnitzii. Complements ANIb values presented in Fig. 4. Dendrogram on top built by hierarchical clustering using Ward.D2 algorithm. (PDF 41 kb)

Additional file 3: Table S1. Strains representing other species of the family Ruminococcaceae, used for comparative purposes in this study. (XLS $17 \mathrm{~kb}$ )

Additional file 4: Table S2. List of 245 orthologous genes in the family Ruminococcaceae used for phylogeny inference. (XLS $534 \mathrm{~kb}$ )

Additional file 5: Figure S3. De novo orthologous protein groups ( $n=10,630$ ) encoded in $31 \mathrm{~F}$. prausnitzii genomes (pangenome). Blue bars, presence of an ortholog; yellow bar, absence of an ortholog. COGannotated orthologous groups are highlighted as black bars on the left. Dendrogram on top built by hierarchical clustering using Ward.D2 algorithm. (PDF $983 \mathrm{~kb}$ )

Additional file 6: Table S3. List of 10,630 protein orthologues encoded by genomes of $F$. prausnitzii with annotation according to COG database. (XLS $915 \mathrm{~kb})$ 
Additional file 7: Figure S4. Composition of F. prausnitzii pan-, core- and accessory genome. A, Pan- and core genome accumulation curves as function of number of included genomes with 100 random permutations. B, Relative size of core- and accessory genome in F. prausnitzii strains. C, Composition of COG categories in the pangenome of $F$. prausnitzii. (PDF $80 \mathrm{~kb}$ )

Additional file 8: Figure S5. Heatmap of gene orthologues differentially abundant between the two F. prausnitzii genomogroups $(p<0.05$ in Wilcoxon test). Dendrogram on top reflect hierarchical clustering using Ward.D2 algorithm. COG annotations for orthologous groups are shown as a colored bar on the left and in the relevant legend inset. Orange colour in heatmap corresponds to single copy orthologues, other colours used for orthologous groups with multiple member per genome (see color code on the right). (PDF $104 \mathrm{~kb}$ )

Additional file 9: Table S4. List of 468 protein orthologues in F. prausnitzil demonstrating differential abundance between the two F. prausnitzii genomogroups. (XLS $58 \mathrm{~kb}$ )

Additional file 10: Table S5. List of prophage regions found in the $F$. prausnitzii genomes and their relationships to the previously descried prophages. (XLS $36 \mathrm{~kb}$ )

Additional file 11: Table S6. Distribution of carbohydrate active enzyme conserved domains in the strains of $F$. prausnitzii. (XLS $35 \mathrm{~kb}$ )

Additional file 12: Figure S6. qPCR analysis of prophage induction in strain F. prausnitzii A2-165. QPCR was performed on DNA extracted from supernatant fractions of overnight A2-165 cultures with or without mitomycin C treatment with primers specific to F. prausnitzii 165 rRNA gene, two complete prophage regions (A2-165_phage_1 and A2165_phage_2corr) and incomplete/remnant prophage. qPCR results were normalized against A2-165 genomic DNA and expressed in arbitrary units (AU) per $\mathrm{mL}$ of culture supernatant. Experiment was performed with 2 independent repeats, each time using 3 biological replicates for each condition. $P$-values calculated using paired t-test. (PDF $19 \mathrm{~kb}$ )

\section{Abbreviations}

ANI: Average nucleotide identity; CD: Crohn's disease; COG: Cluster of orthologous groups; CRC: Colorectal cancer; EOS: Extremely oxygen-sensitive; IBD: Inflammatory bowel disease; ICE: Integrative and conjugative elements; IME: Integrative and mobilizable elements; LCB: Locally collinear blocks; MAM: Microbial anti-inflammatory molecule; pVOG: Prokaryotic virus orthologous groups; ROS: Reactive oxygen species; UC: Ulcerative colitis; VLP: Virus like particle

\section{Acknowledgements}

Authors thank Dr. Amanda Forde (University College Cork), Dr. Lorraine Draper (APC Microbiome Ireland, University College Cork) and Dr. Feargal Ryan (APC Microbiome Ireland, University College Cork) for their insightful discussions.

\section{Funding}

This research was conducted with the financial support of Science Foundation Ireland (SFI) under Grant Number SFI/12/RC/2273, a Science Foundation Ireland's Spokes Programme which is co-funded under the European Regional Development Fund under Grant Number SFI/14/SP APC/ B3032, and a research grant from Janssen Biotech, Inc.

\section{Availability of data and materials}

All genome sequence data used in the analyses were deposited in the NCBI databases under BioProject PRJNA432550 (https://www.ncbi.nlm.nih.gov/ bioproject/432550). Sample IDs, meta data and corresponding accession numbers are summarized in Table 1. Additional raw data tables and $\mathrm{R}$ scripts used for the analysis are available at https://figshare.com/articles/ Comparative_analysis_of_Faecalibacterium_prausnitzii_genomes_shows_ a_high_level_of_genome_plasticity_and_warrants_separation_into_new_ species-level_taxa/7068716.

\section{Authors' contributions}

CBF and ANS conceived and performed the study and drafted the manuscript; TDSS, AVC and W assisted in the analysis of results; RPS and CH secured the funding, provided quidance, participated in drafting and editing of the manuscript. All authors read and approved the final manuscript.

\section{Ethics approval and consent to participate}

Faecal samples were collected from consenting volunteers according to study protocol APC055, approved by the Cork Research Ethics Committee (CREC).

\section{Consent for publication}

Not applicable.

\section{Competing interests}

The authors declare that they have no competing interests.

\section{Publisher's Note}

Springer Nature remains neutral with regard to jurisdictional claims in published maps and institutional affiliations.

\section{Author details}

${ }^{1}$ APC Microbiome Ireland, University College Cork, Cork, Ireland. ${ }^{2}$ Department of Microbiology and Virology, Pirogov Russian National Research Medical University, Moscow, Russia. ${ }^{3}$ Department of Food Biosciences, Teagasc Food Research Centre, Moorepark, Fermoy, Ireland. ${ }^{4}$ School of Microbiology, University College Cork, Cork, Ireland.

Received: 14 September 2018 Accepted: 27 November 2018 Published online: 14 December 2018

\section{References}

1. Cato EP, Salmon CW, Moore WEC. Fusobacterium prausnitzii (Hauduroy et al.) Moore and Holdeman: emended description and designation of Neotype strain. Int J Syst Bacteriol. 1974;24:225-9.

2. Collins MD, Lawson PA, Willems A, Cordoba JJ, Fernandez-Garayzabal J, Garcia P, et al. The phylogeny of the genus Clostridium: proposal of five new genera and eleven new species combinations. Int I Syst Bacteriol. 1994:44:812-26.

3. Duncan SH, Hold GL, Harmsen HJM, Stewart CS, Flint HJ. Growth requirements and fermentation products of fusobacterium prausnitzii, and a proposal to reclassify it as Faecalibacterium prausnitzii gen. Nov., comb. nov. Int J Syst Evol Microbiol. 2002;52:2141-6.

4. Qin J, Li R, Raes J, Arumugam M, Burgdorf KS, Manichanh C, et al. A human gut microbial gene catalogue established by metagenomic sequencing. Nature. 2010;464:59-65.

5. Tap J, Mondot S, Levenez F, Pelletier E, Caron C, Furet J-P, et al. Towards the human intestinal microbiota phylogenetic core. Environ Microbiol. 2009;11: 2574-84.

6. Arumugam M, Raes J, Pelletier E, Le Paslier D, Yamada T, Mende DR, et al. Enterotypes of the human gut microbiome. Nature. 2011;473:174-80.

7. Rajilić-Stojanović M, de Vos WM. The first 1000 cultured species of the human gastrointestinal microbiota. FEMS Microbiol Rev. 2014;38:996-1047.

8. Miquel S, Martín R, Rossi O, Bermúdez-Humarán L, Chatel J, Sokol H, et al. Faecalibacterium prausnitzii and human intestinal health. Curr Opin Microbiol. 2013;16:255-61.

9. Sokol H, Pigneur B, Watterlot L, Lakhdari O, Bermúdez-Humarán LG, Gratadoux J-J, et al. Faecalibacterium prausnitzii is an anti-inflammatory commensal bacterium identified by gut microbiota analysis of Crohn disease patients. Proc Natl Acad Sci. 2008;105:16731-6.

10. Qin J, Li Y, Cai Z, Li S, Zhu J, Zhang F, et al. A metagenome-wide association study of gut microbiota in type 2 diabetes. Nature. 2012;490:55.

11. Borges-Canha M, Portela-Cidade JP, Dinis-Ribeiro M, Leite-Moreira AF, Pimentel-Nunes P. Role of colonic microbiota in colorectal carcinogenesis: a systematic review. Rev Espanola Enfermedades Dig Organo Of Soc Espanola Patol Dig. 2015;107:659-71.

12. Manichanh C, Borruel N, Casellas F, Guarner F. The gut microbiota in IBD. Nat Rev Gastroenterol Hepatol. 2012;9:599.

13. Gevers D, Kugathasan S, Denson LA, Vázquez-Baeza Y, Van Treuren W, Ren $B$, et al. The treatment-naive microbiome in new-onset Crohn's disease. Cell Host Microbe. 2014;15:382-92.

14. Lopez-Siles M, Martinez-Medina M, Abellà C, Busquets D, Sabat-Mir M Duncan SH, et al. Mucosa-associated Faecalibacterium prausnitzii Phylotype richness is reduced in patients with inflammatory bowel disease. Appl Environ Microbiol. 2015;81:7582-92. 
15. Reshef L, Kovacs A, Ofer A, Yahav L, Maharshak N, Keren N, et al. Pouch inflammation is associated with a decrease in specific bacterial taxa. Gastroenterology. 2015;149:718-27.

16. Lopez-Siles M, Martinez-Medina M, Surís-Valls R, Aldeguer X, Sabat-Mir M, Duncan $\mathrm{SH}$, et al. Changes in the abundance of Faecalibacterium prausnitzii Phylogroups I and || in the intestinal mucosa of inflammatory bowel disease and patients with colorectal Cancer. Inflamm Bowel Dis. 2016;22:28-41.

17. Swidsinski A, Loening-Baucke V, Vaneechoutte M, Doerffel Y. Active Crohn's disease and ulcerative colitis can be specifically diagnosed and monitored based on the biostructure of the fecal flora. Inflamm Bowel Dis. 2008;14:147-61.

18. Maccaferri S, Vitali B, Klinder A, Kolida S, Ndagijimana M, Laghi L, et al. Rifaximin modulates the colonic microbiota of patients with Crohn's disease: an in vitro approach using a continuous culture colonic model system. J Antimicrob Chemother. 2010;65:2556-65.

19. Dörffel Y, Swidsinski A, Loening-Baucke V, Wiedenmann B, Pavel M. Common biostructure of the colonic microbiota in neuroendocrine tumors and Crohn's disease and the effect of therapy. Inflamm Bowel Dis. 2012;18:1663-71.

20. Miquel S, Leclerc M, Martin R, Chain F, Lenoir M, Raguideau S, et al. Identification of metabolic signatures linked to anti-inflammatory effects of Faecalibacterium prausnitzii. MBio. 2015;6:e00300-15.

21. Rossi O, Khan MT, Schwarzer M, Hudcovic T, Srutkova D, Duncan SH, et al. Faecalibacterium prausnitzii strain HTF-F and its extracellular polymeric matrix attenuate clinical parameters in DSS-induced colitis. PLoS One. 2015;10:e0123013.

22. Rossi O, van BLA, Chain F, Khan MT, Taverne N, Sokol H, et al. Faecalibacterium prausnitzii A2-165 has a high capacity to induce IL-10 in human and murine dendritic cells and modulates T cell responses. Sci Rep. 2016;6:18507.

23. Quévrain E, Maubert M-A, Sokol H, Devreese B, Seksik P. The presence of the anti-inflammatory protein MAM, from Faecalibacterium prausnitzii, in the intestinal ecosystem. Gut. 2016:65:882.

24. Quévrain E, Maubert MA, Michon C, Chain F, Marquant R, Tailhades J, et al. Identification of an anti-inflammatory protein from Faecalibacterium prausnitzii, a commensal bacterium deficient in Crohn's disease. Gut. 2016;65:415-25.

25. Miquel S, Martín R, Lashermes A, Gillet M, Meleine M, Gelot A, et al. Antinociceptive effect of Faecalibacterium prausnitzii in non-inflammatory IBSlike models. Sci Rep. 2016;6:19399.

26. Tanaka S, Yamamoto K, Yamada K, Furuya K, Uyeno Y. Relationship of enhanced butyrate production by colonic butyrate-producing Bacteria to immunomodulatory effects in Normal mice fed an insoluble fraction of Brassica rapa L. Appl Environ Microbiol. 2016;82:2693-9.

27. Wang H, Jatmiko YD, Bastian SEP, Mashtoub S, Howarth GS. Effects of supernatants from Escherichia coli Nissle 1917 and Faecalibacterium prausnitzii on intestinal epithelial cells and a rat model of 5-fluorouracilinduced mucositis. Nutr Cancer. 2017;69:307-18.

28. Breyner NM, Michon C, Sousa D, S C, Boas V, B P, et al. Microbial antiinflammatory molecule (MAM) from Faecalibacterium prausnitzii shows a protective effect on DNBS and DSS-induced colitis model in mice through inhibition of NF-KB pathway. Front Microbiol. 2017;8. https://doi.org/10. 3389/fmicb.2017.00114.

29. Ohira H, Tsutsui W, Fujioka Y. Are short Chain fatty acids in gut microbiota defensive players for inflammation and atherosclerosis? J Atheroscler Thromb. 2017;24:660-72.

30. Tremaroli $\vee$, Bäckhed F. Functional interactions between the gut microbiota and host metabolism. Nature. 2012;489:242.

31. Lopez-Siles M, Khan TM, Duncan SH, Harmsen HJM, Garcia-Gil LJ, Flint HJ. Cultured representatives of two major Phylogroups of human colonic Faecalibacterium prausnitzii can utilize pectin, Uronic acids, and hostderived substrates for growth. Appl Environ Microbiol. 2012;78:420-8.

32. Hippe B, Remely M, Aumueller E, Pointner A, Magnet U, Haslberger A g. Faecalibacterium prausnitzii phylotypes in type two diabetic, obese, and lean control subjects. Benef Microbes. 2016;7:511-7.

33. Martín R, Miquel S, Benevides L, Bridonneau C, Robert V, Hudault S, et al. Functional characterization of novel Faecalibacterium prausnitzii strains isolated from healthy volunteers: a step forward in the use of F. prausnitzii as a next-generation probiotic. Front Microbiol. 2017;8. https://doi.org/10.3389/fmicb.2017.01226.

34. Benevides L, Burman S, Martin R, Robert V, Thomas M, Miquel S, et al. New insights into the diversity of the genus Faecalibacterium. Front Microbiol. 2017;8. https://doi.org/10.3389/fmicb.2017.01790.

35. Lopez-Siles M, Duncan SH, Garcia-Gil L, Martinez-Medina M. Faecalibacterium prausnitzii: from microbiology to diagnostics and prognostics. ISME J. 2017;11:841.
36. Khan MT, Duncan SH, Stams AJM, van DJM, Flint HJ, Harmsen HJM. The gut anaerobe Faecalibacterium prausnitzii uses an extracellular electron shuttle to grow at oxic-anoxic interphases. ISME J. 2012;6:1578.

37. Heinken A, Khan MT, Paglia G, Rodionov DA, Harmsen HJM, Thiele I. Functional Metabolic Map of Faecalibacterium prausnitzii, a Beneficial Human Gut Microbe. J Bacteriol. 2014;196:3289-302.

38. El-Semman IE, Karlsson FH, Shoaie S, Nookaew I, Soliman TH, Nielsen J. Genomescale metabolic reconstructions of Bifidobacterium adolescentis L2-32 and Faecalibacterium prausnitzii A2-165 and their interaction. BMC Syst Biol. 2014;8:41.

39. Salanitro JP, Muirhead PA, Goodman JR. Morphological and physiological characteristics of Gemmiger formicilis isolated from chicken ceca. Appl Environ Microbiol. 1976;32:623-32.

40. Holmstrøm K, Collins MD, Møller T, Falsen E, Lawson PA. Subdoligranulum variabile gen. nov., sp. nov. from human feces. Anaerobe. 2004;10:197-203.

41. Konstantinidis KT, Ramette A, Tiedje JM. Toward a more robust assessment of Intraspecies diversity, using fewer genetic markers. Appl Environ Microbiol. 2006;72:7286-93.

42. Richter M, Rosselló-Móra R. Shifting the genomic gold standard for the prokaryotic species definition. Proc Natl Acad Sci. 2009;106:19126-31.

43. Li L, Stoeckert CJ, Roos DS. OrthoMCL: Identification of Ortholog groups for eukaryotic genomes. Genome Res. 2003;13:2178-89.

44. Shkoporov AN, Chaplin AV, Shcherbakova VA, Suzina NE, Kafarskaia LI, Bozhenko VK, et al. Ruthenibacterium lactatiformans gen. Nov., sp. nov., an anaerobic, lactate-producing member of the family Ruminococcaceae isolated from human faeces. Int J Syst Evol Microbiol. 2016;66:3041-9.

45. Cornuault JK, Petit M-A, Mariadassou M, Benevides L, Moncaut E, Langella P, et al. Phages infecting Faecalibacterium prausnitzii belong to novel viral genera that help to decipher intestinal viromes. Microbiome. 2018;6:65.

46. Thomas CM, Nielsen KM. Mechanisms of, and barriers to, horizontal gene transfer between Bacteria. Nat Rev Microbiol. 2005:3:711-21.

47. Varghese NJ, Mukherjee S, Ivanova N, Konstantinidis KT, Mavrommatis K, Kyrpides NC, et al. Microbial species delineation using whole genome sequences. Nucleic Acids Res. 2015;43:6761-71.

48. Jia W, Whitehead RN, Griffiths L, Dawson C, Waring RH, Ramsden DB, et al. Is the abundance of Faecalibacterium prausnitzii relevant to Crohn's disease? FEMS Microbiol Lett. 2010;310:138-44.

49. Guédon G, Libante V, Coluzzi C, Payot S, Leblond-Bourget N. The obscure world of integrative and Mobilizable elements, highly widespread elements that pirate bacterial conjugative systems. Genes. 2017;8:337.

50. Zhang Y-X, Perry K, Vinci VA, Powell K, Stemmer WPC, del Cardayré SB. Genome shuffling leads to rapid phenotypic improvement in bacteria. Nature. 2002;415:644-6.

51. Foditsch C, Santos TMA, Teixeira AGV, Pereira RW, Dias JM, Gaeta N, et al. Isolation and characterization of Faecalibacterium prausnitzii from calves and piglets. PLoS One. 2014;9:e116465.

52. Lund M, Bjerrum L, Pedersen K. Quantification of Faecalibacterium prausnitzii- and Subdoligranulum variabile-like bacteria in the cecum of chickens by real-time PCR. Poult Sci. 2010;89:1217-24.

53. Barcenilla A, Pryde SE, Martin JC, Duncan SH, Stewart CS, Henderson C, et al. Phylogenetic relationships of butyrate-producing Bacteria from the human gut. Appl Environ Microbiol. 2000;66:1654-61.

54. Wang RF, Cao WW, Cerniglia CE. PCR detection and quantitation of predominant anaerobic bacteria in human and animal fecal samples. Appl Env Microbiol. 1996;62:1242-7.

55. Shkoporov AN, Efimov BA, Kondova I, Ouwerling B, Chaplin AV, Shcherbakova VA, et al. Peptococcus simiae sp. nov., isolated from rhesus macaque faeces and emended description of the genus Peptococcus. Int J Syst Evol Microbiol. 2016;66:5187-91.

56. Bag S, Ghosh TS, Das B. Complete genome sequence of Faecalibacterium prausnitzii isolated from the gut of a healthy Indian adult. Genome Announc. 2017;5:e01286-17.

57. Bolger AM, Lohse M, Usadel B. Trimmomatic: a flexible trimmer for Illumina sequence data. Bioinformatics. 2014;30:2114-20.

58. Bankevich A, Nurk S, Antipov D, Gurevich AA, Dvorkin M, Kulikov AS, et al. SPAdes: a new genome assembly algorithm and its applications to singlecell sequencing. J Comput Biol. 2012;19:455-77.

59. Aziz RK, Bartels D, Best AA, DeJongh M, Disz T, Edwards RA, et al. The RAST server rapid annotations using subsystems technology. BMC Genomics. 2008;9:75.

60. Pritchard L, Glover RH, Humphris S, Elphinstone JG, Toth IK. Genomics and taxonomy in diagnostics for food security: soft-rotting enterobacterial plant pathogens. Anal Methods. 2015;8:12-24. 
61. Darling ACE, Mau B, Blattner FR, Perna NT. Mauve: multiple alignment of conserved genomic sequence with rearrangements. Genome Res. 2004;14 1394-403.

62. Altschul SF, Madden TL, Schäffer AA, Zhang J, Zhang Z, Miller W, et al. Gapped BLAST and PSI-BLAST: a new generation of protein database search programs. Nucleic Acids Res. 1997;25:3389-402.

63. Edgar RC. MUSCLE: multiple sequence alignment with high accuracy and high throughput. Nucleic Acids Res. 2004;32:1792-7.

64. Galperin MY, Makarova KS, Wolf YI, Koonin EV. Expanded microbial genome coverage and improved protein family annotation in the COG database. Nucleic Acids Res. 2015:43:D261-9.

65. Yin Y, Mao X, Yang J, Chen X, Mao F, Xu Y. dbCAN: a web resource for automated carbohydrate-active enzyme annotation. Nucleic Acids Res. 2012;40:W445-51.

66. Stamatakis A. RAXML version 8: a tool for phylogenetic analysis and postanalysis of large phylogenies. Bioinformatics. 2014;30:1312-3.

67. Arndt D, Grant JR, Marcu A, Sajed T, Pon A, Liang Y, et al. PHASTER: a better, faster version of the PHAST phage search tool. Nucleic Acids Res. 2016;44: W16-21.

68. Roux S, Enault F, Hurwitz BL, Sullivan MB. VirSorter: mining viral signal from microbial genomic data. PeerJ. 2015;3:e985.

69. Grazziotin AL, Koonin EV, Kristensen DM. Prokaryotic virus orthologous groups (pVOGs): a resource for comparative genomics and protein family annotation. Nucleic Acids Res. 2017:45:D491-8.

70. Shkoporov AN, Ryan FJ, Draper LA, Forde A, Stockdale SR, Daly KM, et al. Reproducible protocols for metagenomic analysis of human faecal phageomes. Microbiome. 2018;6:68.

71. Langmead B, Salzberg SL. Fast gapped-read alignment with bowtie 2. Nat Methods. 2012;9:357-9.

72. Suzuki R, Shimodaira H. Pvclust: an R package for assessing the uncertainty in hierarchical clustering. Bioinformatics. 2006;22:1540-2.

73. Browne HP, Forster SC, Anonye BO, Kumar N, Neville BA, Stares MD, et al. Culturing of 'unculturable' human microbiota reveals novel taxa and extensive sporulation. Nature. 2016;533:543-6.

Ready to submit your research? Choose BMC and benefit from:

- fast, convenient online submission

- thorough peer review by experienced researchers in your field

- rapid publication on acceptance

- support for research data, including large and complex data types

- gold Open Access which fosters wider collaboration and increased citations

- maximum visibility for your research: over $100 \mathrm{M}$ website views per year

At $\mathrm{BMC}$, research is always in progress.

Learn more biomedcentral.com/submissions 